\title{
MIXED TÊTE-À-TÊTE TWISTS AS MONODROMIES ASSOCIATED WITH HOLOMORPHIC FUNCTION GERMS
}

\author{
PABLO PORTILLA CUADRADO AND BALDUR SIGURĐSSON
}

\begin{abstract}
Tête-à-tête graphs were introduced by N. A'Campo in 2010 with the goal of modeling the monodromy of isolated plane curves. Mixed têteà-tête graphs provide a generalization which define mixed tête-à-tête twists, which are pseudo-periodic automorphisms on surfaces. We characterize the mixed tête-à-tête twists as those pseudo-periodic automorphisms that have a power which is a product of right-handed Dehn twists around disjoint simple closed curves, including all boundary components. It follows that the class of tête-à-tête twists coincides with that of monodromies associated with reduced function germs on isolated complex surface singularities. Finally, using the language of plumbing calculus, we relate horizontal open book decompositions of graph manifolds with mixed tête-à-tête graphs via two algorithms.
\end{abstract}

\section{CONTENTS}

1. Introduction

2. The mapping class group

3. Pseudo-periodic automorphisms

4. Pure tête-à-tête graphs

5. Mixed tête-à-tête graphs

6. Realization theorem

7. Horizontal open books in graph manifolds

8. Description of the monodromy

9. From horizontal open books to mixed tête-à-tête graphs 25

10. From mixed tête-à-tête graphs to open books 26

11. Examples

References

\section{INTRODUCTION}

In $\left[\mathrm{A}^{\prime} \mathrm{C}\right] \mathrm{N}$. A'Campo introduced the notion of pure tête-à-tête graph in order to model monodromies of plane curves. These are metric ribbon graphs without

Date: April 13, 2018.

First author supported by SVP-2013-067644 Severo Ochoa FPI grant and by project by MTM2013-45710-C2-2-P, the two of them by the Spanish Ministry of Economy and Competitiveness MINECO; also supported by the project ERCEA 615655 NMST Consolidator Grant.

The second author is supported by the ERCEA 615655 NMST Consolidator Grant, by the Basque Government through the BERC 2014-2017 program and by the Spanish Ministry of Economy and Competitiveness MINECO: BCAM Severo Ochoa excellence accreditation SEV-20130323. 
univalent vertices that satisfy a special property. If one sees the ribbon graph $\Gamma$ as a strong deformation retract of a surface $\Sigma$ with boundary, the tête-à-tête propety says that starting at any point $p$ and walking a distance of $\pi$ in any direction and always turning right at vertices, gets you to the same point. This property defines an element in the mapping class group $\mathrm{MCG}^{+}(\Sigma, \partial \Sigma)$ which is freely periodic, and is called the tête-à-tête twist associated with $\Gamma$.

In [Gra14], C. Graf proved that if one allows univalent vertices in tête-à-tête graphs, then one is able to model all freely periodic mapping classes of $\mathrm{MCG}^{+}(\Sigma, \partial \Sigma)$ with positive fractional Dehn twist coefficients. In [FPP] this result was improved by showing that one does not need to enlarge the original class of metric ribbon graphs used to prove the same theorem.

However, the geometric monodromy of an isolated plane curve singularity with one branch and more than one Puiseux pair is not of finite order (see [ $\left.\mathrm{A}^{\prime} \mathrm{C} 73\right]$ ). For this purpose, the definition of mixed tête-à-tête graph was introduced in [FPP]. These are metric ribbon graphs together with a filtration and a set of locally constant functions that model a class of pseudo-periodic automorphisms. It was proved that this class includes the monodromy associated with an isolated singularity on a plane curve with one branch.

In this work we continue the study of (relative) mixed tête-à-tête graphs and we improve and generalize results from $[\mathrm{FPP}]$ and $[\mathrm{Por}]$. The main result is Theorem 6.7 which says

Theorem A. Let $\phi: \Sigma \rightarrow \Sigma$ be an automorphism with fixes the boundary. Then there exists a mixed tête-à-tête graph in $\Sigma$ inducing its mapping class in $\mathrm{MCG}^{+}(\Sigma, \partial \Sigma)$ if and only if some power of $\phi$ is a composition of right handed Dehn twists around disjoint simple closed curves including all boundary components.

A reduced holomorphic function germ on an isolated surface singularity has an associated Milnor fibration with a monodromy which fixes the boundary. It is known that this monodromy is pseudo-periodic and a power of it is a composition of right-handed Dehn twists around disjoint simple closed curves, which include all the boundary curves. It follows that the Milnor fiber associated with such a function germ contains a mixed tête-à-tête graph which defines the monodromy. Conversely, a result by Neumann and Pichon [NP07] says that any such a surface automorphism is realized as the monodromy associated with a function germ. Hence

Theorem B. Mixed tête-à-tête twists are precisely the monodromies associated with reduced function germs on isolated surface singularities.

The mapping torus of a pseudo-periodic surface automorphism is a graph manifold. Conversely, a horizontal surface of a fiber-oriented graph manifold has a pseudo-periodic monodromy induced on it. Hence, it is a natural problem to assign a graph manifold and a horizontal surface to each mixed tête-à-tête graph and, whenever possible, assign a mixed tête-à-tête graph to a given horizontal surface in a graph manifold. In the second part of the article we start by reviewing known results and definitions related to graph manifolds and their fibrations over $S^{1}$ (for more details on this topic see [Pic01, Neu81, LP05, EN85, Neu97, Wal67]). We then provide algorithms producing a graph manifold and a fibration over $S^{1}$ from a mixed tête-à-tête graph and vice versa. In particular, this provides a direct and effective way to check if two mixed tête-à-tête graphs represent conjugate mapping classes in $\mathrm{MCG}^{+}(\Sigma, \partial \Sigma)$. 
The structure of the work is the following. In Section 2 we briefly introduce notation related to the mapping class group that we use throughout the text.

In Section 3 we fix notation and conventions about pseudo-periodic automorphisms of surfaces. Not all of this section is contained in [FPP] since we treat a broader class of pseudo-periodic automorphisms in the present text. In particular we allow amphidrome orbits of annuli. This section is important for Section 6 which contains the main results.

In Sections 4 and 5 we recall some necessary definitions and results about pure and mixed tête-à-tête graphs.

Section 6 starts with the statement of the main Theorem 6.7. Two Lemmas which are used in the proof follow and the section ends with the proof of the main result.

As an application of the main result, we understand the relation between horizontal open books of graph manifolds and mixed tête-à-tête graphs.

In Proposition 7.10 we state a classification result for horizontal fibrations of the complement of a link in a graph manifold by a certain subset of the first cohomology of the graph manifold. The proof of this classification is constructive and essentially follows [EN85]. The line of argument presented is useful for Sections 9 and 10.

In Section 8 we explain how to obtain the combinatorial data that determines a pseudo-periodic automorphism up to isotopy and conjugation from a horizontal open book.

Finally, in Sections 9 and 10 we detail the algorithms to travel from horizontal open books to pseudo-periodic automorphisms and vice versa.

The work ends with three examples in Section 11 that show how the main theorem and the two algorithms are applied to particular cases.

\section{The MAPPING ClASS GROUP}

2.1. Let $\Sigma$ be a surface with $\partial \Sigma \neq \emptyset$. We denote by $\operatorname{MCG}(\Sigma)$ the mapping class group given by the automorphisms of $\Sigma$ up to isotopy, where the automorphisms of the isotopy do not neccesarily fix the boundary. Let $\partial^{1} \Sigma \subset \partial \Sigma$ be a subset formed by some boundary components of $\Sigma$. We will denote by $\operatorname{MCG}\left(\Sigma, \partial^{1} \Sigma\right)$ the mapping class group given by automorphisms of $\Sigma$ that are the identity restricted to $\partial^{1} \Sigma$ and where isotopies are along automorphisms that are the identity on these boundary components.

Let $\phi: \Sigma \rightarrow \Sigma$ be a automorphism, we denote its class in $\operatorname{MCG}(\Sigma)$ by $[\phi]$. If $\left.\phi\right|_{\partial^{1} \Sigma}=$ id we denote its class in $\operatorname{MCG}\left(\Sigma, \partial^{1} \Sigma\right)$ by $[\phi]_{\partial^{1} \Sigma}$.

Given two automorphisms $\phi$ and $\psi$ of $\Sigma$ that both leave invariant some subset $B \subset \partial \Sigma$ such that $\left.\phi\right|_{B}=\left.\psi\right|_{B}$, we say they are isotopic relative to the action $\left.\phi\right|_{B}$ if there exists a family of automorphisms of $\Sigma$ that isotope them as before and such that any automorphism of the family has the same restriction to $B$ as $\phi$ and $\psi$. We write $[\phi]_{B,\left.\phi\right|_{B}}=[\psi]_{B,\left.\phi\right|_{B}}$. We denote by $\operatorname{MCG}\left(\Sigma, B,\left.\phi\right|_{B}\right)$ the set of classes $[\phi]_{B,\left.\phi\right|_{B}}$ with respect to this equivalence relation. We denote by $\operatorname{MCG}^{+}\left(\Sigma, B,\left.\phi\right|_{B}\right)$ if we restrict to automorphisms preserving orientation.

2.2. Consider an automorphism $\phi: \Sigma \rightarrow \Sigma$ with $\left.\phi\right|_{\partial^{1} \Sigma}=$ id for some subset $\partial^{1} \Sigma \subset \partial \Sigma$. Let $\mathcal{D}_{i}$ denote a right-handed Dehn twist around a curve parallel to the boundary component $C_{i} \subset \partial^{1} \Sigma$. Suppose that $[\phi] \in \operatorname{MCG}(\Sigma)$ is of finite order, i.e. there exists a natural number $n$ such that $[\phi]^{n}=[\mathrm{id}]$. Then we have that 
$\left[\phi^{n}\right]_{\partial^{1} \Sigma}=\left[\mathcal{D}_{1}\right]_{\partial^{1} \Sigma}^{t_{1}} \cdots\left[\mathcal{D}_{r}\right]_{\partial^{1} \Sigma}^{t_{r}}$ with $t_{i} \in \mathbb{Z}$. We call $t_{i} / n$ the fractional Dehn twist coefficient of $\phi$ at the component $C_{i}$.

\section{Pseudo-Periodic Automorphisms}

We recall conventions, definitions and results from [FPP] Part II. that we will use in the present work. We also extend some of the notions there to cover some cases that were not treated in that work.

Definition 3.1. A automorphism $\phi: \Sigma \rightarrow \Sigma$ is pseudo-periodic if it is isotopic to a automorphism satisfying that there exists a finite collection of disjoint simple closed curves $\mathcal{C}$ such that

(i) $\phi(\mathcal{C})=\mathcal{C}$.

(ii) $\left.\phi\right|_{\Sigma \backslash \mathcal{C}}$ is freely isotopic to a periodic automorphism.

Assuming that none of the connected components of $\Sigma \backslash \mathcal{C}$ is either a disk or an annulus and that the set of curves is minimal, which is always possible, we name $\mathcal{C}$ an admissible set of curves for $\phi$.

The following theorem is a particularization on pseudo-periodic automorphisms of the more general Corollary 13.3 in [FM12] that describes a canonical form for every automorphism of a surface.

Theorem 3.2 (Almost-Canonical Form and Canonical Form). Let $\Sigma$ be a surface with $\partial \Sigma \neq \emptyset$. Any pseudo-periodic map of $\Sigma$ is isotopic to an automorphism in almost-canonical form, that means a automorphism $\phi$ which has an admissible set of curves $\mathcal{C}=\left\{\mathcal{C}_{i}\right\}$ and annular neighborhoods $\mathcal{A}=\left\{\mathcal{A}_{i}\right\}$ with $\mathcal{C}_{i} \subset \mathcal{A}_{i}$ such that

(i) $\phi(\mathcal{A})=\mathcal{A}$.

(ii) The map $\left.\phi\right|_{\overline{\Sigma \backslash \mathcal{A}}}$ is periodic.

When the set $\mathcal{C}$ is minimal we say that $\phi$ is in canonical form.

Remark 3.3. In the case we have a pseudo-periodic automorphism of $\Sigma$ that fixes pointwise some components $\partial^{1} \Sigma$ of the boundary $\partial \Sigma$ we can always find $a$ canonical form as follows. We can find an isotopic automorphism $\phi$ relative to $\partial^{1} \Sigma$ that coincides with a canonical form as in the previous theorem outside a collar neighborhood $U$ of $\partial^{1} \Sigma$. We may assume that there exists an isotopy connecting the automorphism and its canonical form relative to $\partial^{1} \Sigma$.

3.4. Let $\phi$ be a pseudo-periodic automorphism in some almost-canonical form. Let $\mathcal{C}_{1}, \ldots, \mathcal{C}_{k}$ be a subset of curves in $\mathcal{C}$ that are cyclically permuted by $\phi$, i.e. $\phi\left(\mathcal{C}_{i}\right)=\mathcal{C}_{i+1}$ for $i=1, \ldots, k-1$ and $\phi\left(\mathcal{C}_{k}\right)=C_{1}$. Suppose that we give an orientation to $\mathcal{C}_{1}, \ldots, \mathcal{C}_{k}$ so that $\left.\phi\right|_{\mathcal{C}_{i}}$ for $i=1, \ldots, k-1$ is orientation preserving. We say that the curves are amphidrome if $\left.\phi\right|_{\mathcal{C}_{k}}: \mathcal{C}_{k} \rightarrow \mathcal{C}_{1}$ is orientation reversing.

Notation 3.5. Let $s, c \in \mathbb{R}$. We denote by $\mathcal{D}_{s, c}$ the automorphism of $\mathbb{S}^{1} \times I$ given by by $(x, t) \mapsto(x+s t+c, t)$ (we are taking $\mathbb{S}^{1}=\mathbb{R} / \mathbb{Z}$ ). Observe that

$$
\begin{gathered}
\mathcal{D}_{s, c} \circ \mathcal{D}_{s^{\prime}, c^{\prime}}=\mathcal{D}_{s+s^{\prime}, c+c^{\prime}}, \\
\mathcal{D}_{s, c}^{-1}=\mathcal{D}_{-s,-c} .
\end{gathered}
$$

In this work we always have $s \in \mathbb{Q}$. 
Remark 3.6. We can isotope $\mathcal{D}_{s, c}$ to a automorphism $\mathcal{D}_{s, c}^{p}$ that is periodic on some tubular neighborhood of the core curve $\mathbb{S}^{1} \times\{1 / 2\}$ of the annulus while preserving the action on the boundary $\partial\left(\mathbb{S}^{1} \times I\right)$ :

$$
\mathcal{D}_{s, c}^{p}(x, t)= \begin{cases}\left(x+3 s\left(t-\frac{1}{3}\right)+c, t\right) & 0 \leq t \leq \frac{1}{3} \\ (x+c, t) & \frac{1}{3} \leq t \leq \frac{2}{3} \\ \left(x+3 s\left(t-\frac{2}{3}\right)+c, t\right) & \frac{2}{3} \leq t \leq 1\end{cases}
$$

Notation 3.8. We denote by $\tilde{\mathcal{D}}_{s}$ the automorphism of $\mathbb{S}^{1} \times I$ given by

$$
\tilde{\mathcal{D}}_{s}(x, t)= \begin{cases}\left(-x-3 s\left(t-\frac{1}{3}\right), 1-t\right) & 0 \leq t \leq \frac{1}{3} \\ (-x, 1-t) & \frac{1}{3} \leq t \leq \frac{2}{3} \\ \left(-x-3 s\left(t-\frac{2}{3}\right), 1-t\right) & \frac{2}{3} \leq t \leq 1\end{cases}
$$

In this case we only work with $s \in \mathbb{Q}$ as well.

Definition 3.10. Let $\mathcal{C} \subset \Sigma$ be a simple closed curve embedded in an oriented surface $\Sigma$. And let $\mathcal{A}$ be a tubular neighborhood of $\mathcal{C}$. Let $\mathcal{D}: \Sigma \rightarrow \Sigma$ be a automorphism of the surface with $\left.\mathcal{D}\right|_{\Sigma \backslash \mathcal{A}}=\mathrm{id}$. We say that $\mathcal{D}$ is a negative Dehn twist around $\mathcal{C}$ or a right-handed Dehn twist if there exist a parametrization $\eta$ : $\mathbb{S}^{1} \times I \rightarrow \mathcal{A}$ preserving orientation such that

$$
\mathcal{D}=\eta \circ \mathcal{D}_{1,0} \circ \eta^{-1} .
$$

A positive Dehn twist is defined similarly changing $\mathcal{D}_{1,0}$ by $\mathcal{D}_{-1,0}$ in the formula above.

Lemma 3.11 (Linearization. Equivalent to Lemma 2.1 in [MM11]). Let $\mathcal{A}_{i}$ be an annulus and let $\phi: \mathcal{A}_{i} \rightarrow \mathcal{A}_{i}$ be a automorphism that does not exchange boundary components. Suppose that $\left.\phi\right|_{\partial \mathcal{A}_{i}}$ is periodic. Then, after an isotopy fixing the boundary, there exists a parametrization $\eta: \mathbb{S}^{1} \times I \rightarrow \mathcal{A}_{i}$ such that

$$
\phi=\eta \circ \mathcal{D}_{-s,-c} \circ \eta^{-1}
$$

for some $s \in \mathbb{Q}$, some $c \in \mathbb{R}$.

Lemma 3.12 (Specialization. Equivalent to Lemma 2.3 in [MM11]). Let $\mathcal{A}_{i}$ be an annulus and let $\phi: \mathcal{A}_{i} \rightarrow \mathcal{A}_{i}$ be a automorphism that exchanges boundary components. Suppose that $\left.\phi\right|_{\partial \mathcal{A}_{i}}$ is periodic. Then after an isotopy fixing the boundary, there exists a parametrization $\eta: \mathbb{S}^{1} \times I \rightarrow \mathcal{A}_{i}$ such that

$$
\phi=\eta \circ \tilde{\mathcal{D}}_{-s} \circ \eta^{-1}
$$

for some $s \in \mathbb{Q}$.

Remark 3.13. In the case $\left.\phi\right|_{\partial \mathcal{A}_{i}}$ is the identity, we have that

$$
\phi=\eta \circ \mathcal{D}_{s, 0} \circ \eta^{-1}
$$

for some $s \in \mathbb{Z}$, that is $\phi=\mathcal{D}^{s}$ for some right-handed Dehn twist as in Definition 3.10 .

Definition 3.14 (Screw number). Let $\phi$ be a pseudo-periodic automorphism as in Theorem 3.2. Let $n$ be the order of $\left.\phi\right|_{\Sigma \backslash \mathcal{A}}$. By Remark 3.13, $\left.\phi^{n}\right|_{\mathcal{A}_{i}}$ equals $\left.\mathcal{D}\right|_{\mathcal{A}_{i}} ^{s_{i}}$ for a certain $s_{i} \in \mathbb{Z}$. 
Let $\alpha$ be the length of the orbit in which $\mathcal{A}_{i}$ lies and let $\tilde{\alpha} \in\{\alpha, 2 \alpha\}$ be the smallest number such that $\phi^{\tilde{\alpha}}$ does not exchange the boundary components of $\mathcal{A}_{1}$. We define

$$
s\left(\mathcal{A}_{i}\right):=\frac{-s_{i}}{n} \tilde{\alpha}
$$

We call $s\left(\mathcal{A}_{i}\right)$ the screw number of $\phi$ at $\mathcal{A}_{i}$ or at $\mathcal{C}_{i}$.

Remark 3.15. Compare Definition 3.14 with [[MM11] p.4] and with Definition 2.4 in [MM11]. The original definition is due to Nielsen [[Nie44]. Sect. 12] and it does not depend on a canonical form for $\phi$. Since we are restricting to automorphisms that do not exchange boundary components of the annuli $\mathcal{A}$, our definition is a bit simpler.

Lemma 3.16. Let $\phi$ be a automorphism as in Theorem 3.2 and let $\left\{\mathcal{A}_{i}\right\} \subset \mathcal{A}$ be a set of $k$ annuli cyclically permuted by $\phi$, i.e. $\phi\left(\mathcal{A}_{i}\right)=\mathcal{A}_{i+1}$ such that $\phi^{k}$ does not exchange boundary components. Then there exist coordinates

$$
\eta_{i}: \mathbb{S}^{1} \times I \rightarrow \mathcal{A}_{i}
$$

for the annuli in the orbit such that

$$
\eta_{j+1}^{-1} \circ \phi \circ \eta_{j}=\mathcal{D}_{-s / k,-c / k}
$$

where $s$ and $c$ are associated to $\mathcal{A}_{1}$ as in Lemma 3.11 .

Proof. See [FPP]

Remark 3.17. By Remark 3.6 we can substitute $\mathcal{D}_{-s / k,-c / k}$ by $\mathcal{D}_{-s / k,-c / k}^{p}$ in the previous lemma.

Lemma 3.18. Let $\phi$ be a automorphism as in Theorem 3.2 and let $\left\{\mathcal{A}_{i}\right\} \subset \mathcal{A}$ be a set of $k$ annuli cyclically permuted by $\phi$, i.e. $\phi\left(\mathcal{A}_{i}\right)=\mathcal{A}_{i+1}$ such that $\phi^{k}$ exchanges boundary components. Then there exist coordinates

$$
\eta_{i}: \mathbb{S}^{1} \times I \rightarrow \mathcal{A}_{i}
$$

for the annuli in the orbit such that

$$
\eta_{j+1}^{-1} \circ \phi \circ \eta_{j}=\tilde{\mathcal{D}}_{-s / \alpha}
$$

where $s$ is associated to $\mathcal{A}_{1}$ as in Lemma 3.12.

Proof. Take a parametrization of $\mathcal{A}_{1}$ for $\phi^{k}: \mathcal{A}_{1} \rightarrow \mathcal{A}_{1}$ as in Lemma 3.12, say $\eta_{1}: \mathbb{S}^{1} \times I \rightarrow \mathcal{A}_{1}$. Define recursively $\eta_{j}:=\phi \circ \eta_{j-1} \circ \tilde{\mathcal{D}}_{s / k}$ (see Notation 3.5 ). Then, we have

$$
\eta_{j+1}^{-1} \circ \phi \circ \eta_{j}=\tilde{\mathcal{D}}_{-s / k}
$$

Since for every $j$ we have that $\eta_{j}=\phi^{j-1} \circ \eta_{1} \circ \tilde{\mathcal{D}}_{s(j-1) / k}$ we have also that

$$
\eta_{1}^{-1} \circ \phi \circ \eta_{\alpha}=\eta_{1}^{-1} \circ \phi \circ \phi^{k-1} \circ \eta_{1} \circ \tilde{\mathcal{D}}_{s(k-1) / k}=\tilde{\mathcal{D}}_{-s / k}
$$

Remark 3.19. After this proof we can check that $\eta_{k}^{-1} \circ \phi^{\alpha} \circ \eta_{k}=\mathcal{D}_{-s,-c}$ to see that the screw number $s=s\left(\mathcal{A}_{i}\right)$ and the parameter $c$ modulo $\mathbb{Z}$ of Lemma 3.16 only depend on the orbit of $\mathcal{A}_{i}$.

We observe also that the numbers $s$ and $c$ of Lemma 3.16 satisfy

$s$ equals $s\left(\mathcal{A}_{i}\right)$ and 
S $c$ is only determined modulo $\mathbb{Z}$ and equals the usual rotation number $\operatorname{rot}\left(\left.\phi^{\alpha_{i}}\right|_{\eta\left(\mathbb{S}^{1} \times\{0\}\right)}\right) \in(0,1]$.

This is also observed in Corollary 2.2 in [MM11].

Definition 3.20. Let $C$ be a component of $\partial \Sigma$ and let $\mathcal{A}$ be a compact collar neighborhood of $C$ in $\Sigma$. Suppose that $C$ has a metric and total length is equal to $\ell$. Let $\eta: \mathbb{S}^{1} \times I \rightarrow \mathcal{A}$ be a parametrization of $\mathcal{A}$, such that $\left.\eta\right|_{\mathbb{S}^{1} \times\{1\}}: \mathbb{S}^{1} \times\{1\} \rightarrow C$ is an isometry.

Suppose that $\mathbb{S}^{1}$ has the metric induced from taking $\mathbb{S}^{1}=\mathbb{R} / \ell \mathbb{Z}$ with $\ell \in \mathbb{R}_{>0}$ and the standard metric on $\mathbb{R}$. A boundary Dehn twist of length $r \in \mathbb{R}_{>0}$ along $C$ is a automorphism $\mathcal{D}_{r}^{\eta}(C)$ of $\Sigma$ such that:

(i) it is the identity outside $\mathcal{A}$

(ii) the restriction of $\mathcal{D}_{r}^{\eta}(C)$ to $\mathcal{A}$ in the coordinates given by $\eta$ is given by $(x, t) \mapsto(x+r \cdot t, t)$.

The isotopy type of $\mathcal{D}_{r}^{\eta}(C)$ by isotopies fixing the action on $\partial \Sigma$ does not depend on the parametrization $\eta$. When we write just $\mathcal{D}_{r}(C)$, it means that we are considering a boundary Dehn twist with respect to some parametrization $\eta$.

Remark 3.21. Given a automorphism $\phi$ of a surface $\Sigma$ with $\partial \Sigma \neq \emptyset$. Let $C$ be a connected component of $\partial \Sigma$ such that $\left.\phi\right|_{C}$ is a rotation by $c \in[0,1)$. Let $\mathcal{A}$ be a compact collar neighborhood of $C$ (isomorphic to $I \times C$ ) in $\Sigma$. Let $\eta: \mathbb{S}^{1} \times I \rightarrow \mathcal{A}$ be a parametrization of $\mathcal{A}$, with $\phi\left(\mathbb{S}^{1} \times\{1\}\right)=C$. Up to isotopy, we can assume that the restriction of $\phi$ to $\mathcal{A}$ satisfies

$$
\left.\eta^{-1} \circ \phi\right|_{\mathcal{A}} \circ \eta(x, t)=(x+c, t) .
$$

\section{Pure tÊTE-À-tête GraPhS}

In this section we recall some definitions and conventions from [FPP] Part I.

4.1. A graph $\Gamma$ is a 1 dimensional finite $C W$-complex; unless otherwise specified a graph doesn't have univalent vertices. A ribbon graph is a graph equipped with a cyclic ordering of the edges adjacent to each vertex. With a ribbon graph, one can recover the topology of an orientable surface with boundary, we call this surface the thickening of $\Gamma$. A metric ribbon graph is a ribbon graph with a metric on each of its edges.

A relative metric ribbon graph is a pair $(\Gamma, A)$ with $A \subset \Gamma$ a subgraph formed by a disjoint union of circles with the property that for each connected component $A_{i} \subset A$, there exists a boundary component on the thickening $\Sigma$ of $\Gamma$ such that it retracts to $A_{i}$. The relative thickening of $(\Gamma, A)$ is the thickening of $\Gamma$ minus the cylinders corresponding to the boundary components that retract to $A$. In particular, the relative thickening, also denoted by $\Sigma$ contains $A$ as boundary.

Definition 4.2 (Safe walk). Let $(\Gamma, A)$ be a metric relative ribbon graph. A safe walk for a point $p$ in the interior of some edge is a path $\gamma_{p}: \mathbb{R}_{\geq 0} \rightarrow \Gamma$ with $\gamma_{p}(0)=p$ and such that:

(1) The absolute value of the speed $\left|\gamma_{p}^{\prime}\right|$ measured with the metric of $\Gamma$ is constant and equal to 1 . Equivalently, the safe walk is parametrized by arc length, i.e. for $s$ small enough $d\left(p, \gamma_{p}(s)\right)=s$.

(2) when $\gamma_{p}$ gets to a vertex, it continues along the next edge in the given cyclic order. 
(3) If $p$ is in an edge of $A$, the walk $\gamma_{p}$ starts running in the opposite direction to the one indicated by $A$ seen as boundary of $\Sigma$.

An $\ell$-safe walk is the restriction of a safe walk to the interval $[0, \ell]$. If a length is not specified when referring to a safe walk, we will understand that its length is $\pi$.

The notion in (2) of continuing along the next edge in the order of $e(v)$ is equivalent to the notion of turning to the right in every vertex for paths parallel to $\Gamma$ in $\Sigma$ in $\mathrm{A}^{\prime}$ Campo's words in $\left[\mathrm{A}^{\prime} \mathrm{C}\right]$.

Definition 4.3 (Tête-à-tête property). Let $(\Gamma, A)$ be relative metric ribbon graph. We say that $\Gamma$ satisfies the $\ell$-tête-à-tête property, or that $\Gamma$ is an $\ell$-tête-à-tête graph if

1) For any point $p \in \Gamma \backslash(A \cup v(\Gamma))$ the two different $\ell$-safe walks starting at $p$, that we denote by $\gamma_{p}, \omega_{p}$, satisfy $\gamma_{p}(\ell)=\omega_{p}(\ell)$.

2) for a point $p$ in $A \backslash v(\Gamma)$, the end point of the unique $\ell$-safe walk starting at $p$ belongs to $A$.

In this case, we say that $(\Gamma, A)$ is a relative $\ell$-tête-à-tête graph. If $A=\emptyset$, we call it a pure $\ell$-tête-à-tête structure or graph. If $\ell=\pi$ we just call it pure tête-à-tête structure or graph.
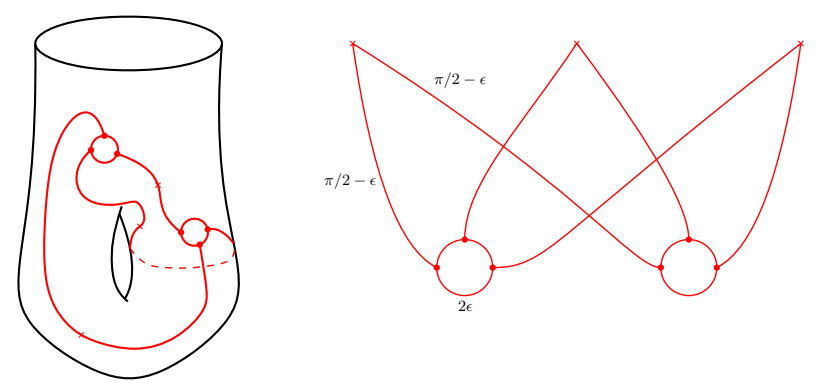

Figure 4.1. An example of a relative tête-à-tête graph. It has 2 connected components in $A$ (the two small circles). The length of an edge in $A$ is $2 \varepsilon$ and the length of and edge from a vertex in $A$ to a vertex depicted as a cross is $\pi / 2-\varepsilon$.

Notation 4.4. Let $(\Gamma, A) \hookrightarrow(\Sigma, \partial \Sigma)$ be a relative ribbon graph properly embedded in its thickening. Let $\Sigma_{\Gamma}$ be the surface that results from cutting $\Sigma$ along $\Gamma$, then $\Sigma_{\Gamma}$ consists of as many cylinders as there are connected components in $\partial \Sigma \backslash A$. We denote these cylinders by $\tilde{\Sigma}_{1}, \ldots, \tilde{\Sigma}_{r}$.

Let $g_{\Gamma}: \Sigma_{\Gamma} \rightarrow \Sigma$ be the gluing map. We denote by $\widetilde{\Gamma}_{i}$ the boundary component of the cylinder $\Sigma_{i}$ that comes from the graph (that is $g_{\Gamma}\left(\widetilde{\Gamma}_{i}\right) \subset \Gamma$ ) and by $C_{i}$ the one coming from a boundary component of $\Sigma$ (that is $g_{\Gamma}\left(C_{i}\right) \subset \partial \Sigma$ ). From now on, we take the convention that $C_{i}$ is identified with $C_{i} \times\{1\}$ and that $\tilde{\Gamma}_{i}$ is identified with $C_{i} \times\{0\}$. We set $\Sigma_{i}:=g_{\Gamma}\left(\widetilde{\Sigma}_{i}\right)$ and $\Gamma_{i}:=g_{\Gamma}\left(\widetilde{\Gamma}_{i}\right)$. Finally we denote $g_{\Gamma}\left(C_{i}\right)$ also by $C_{i}$ since $\left.g_{\Gamma}\right|_{C_{i}}$ is bijective.

A retraction or a product structure for a component $\Sigma_{i}$ is a parametrization

$$
r_{i}: \mathbb{S}^{1} \times I \rightarrow \Sigma_{i} .
$$

For each $\theta \in \mathbb{S}^{1}$, we call $r_{i}(\{\theta\} \times I)$ a retraction line. We also say that $g_{\Gamma}\left(r_{i}(\{\theta\} \times I)\right)$ is a retraction line. 
4.5. A relative tête-à-tête graph $(\Gamma, A) \hookrightarrow(\Sigma, \partial A)$ induces a mapping class $\left[\phi_{\Gamma}\right]_{\partial \Sigma \backslash A \text {,id }}$ on $\Sigma$, more specifically, an element of $\operatorname{MCG}(\Sigma, \partial \Sigma \backslash A)$. If a product structure is specified, then an explicit representative $\phi_{\Gamma}$ is induced. For any product structure $\phi_{\Gamma}$ satisfies:

1) $\left.\phi_{\Gamma}\right|_{\Gamma}(p)=\gamma_{p}(\pi)$, that is, it induces on the graph the same action as the tête-àtête property.

2) the mapping class $\left[\phi_{\Gamma}\right] \in \operatorname{MCG}(\Sigma)$ is of finite order, we also say it is periodic.

3 ) the fractional Dehn twist coefficients $t_{i} / n$ (recall 2.2) along all boundary components in $\Sigma \backslash A$ are strictly positive.

Actually, in [FPP] it is proven that 2) and 3) above characterize tête-à-tête automorphisms, more concretely the following is proven:

Theorem 4.6. Let $\phi: \Sigma \rightarrow \Sigma$ be an automorphism of a surface with $\left.\phi\right|_{\partial^{1} \Sigma}=\mathrm{id}$ for some non-empty subset $\partial^{1} \Sigma \subset \partial \Sigma$. Then there exists a relative tête-à-tête graph $\left(\Gamma, \partial \Sigma \backslash \partial^{1} \Sigma\right)$ with $\left[\phi_{\Gamma}\right]_{\partial^{1} \Sigma}=[\phi]_{\partial^{1} \Sigma}$ if and only if $[\phi] \in \operatorname{MCG}(\Sigma)$ is of finite order and all the fractional Dehn twists at boundary components in $\partial^{1} \Sigma$ are strictly positive.

It corresponds to Theorem 9.12 in [FPP]. Actually it is a consequence of that Theorem since there, the authors consider also negative fractional Dehn twists and also negative safe walks (which turn left instead of turning right). This is done via a sign map $\iota: \partial^{1} \Sigma \rightarrow\{+,-, 0\}$.

In this work we only use the original notion of A'Campo, so this map is constant + .

\section{MIXED TÊTE-À-TÊTE GRAPHS}

With pure, relative and general tête-à-tête graphs we model periodic automorphisms. Now we extend the notion of tête-à-tête graph to be able to model some pseudo-periodic automorphisms.

Let $\left(\Gamma^{\bullet}, A^{\bullet}\right)$ be a decreasing filtration on a connected relative metric ribbon graph $(\Gamma, A)$. That is

$$
(\Gamma, A)=\left(\Gamma^{0}, A^{0}\right) \supset\left(\Gamma^{1}, A^{1}\right) \supset \cdots \supset\left(\Gamma^{d}, A^{d}\right)
$$

where $\supset$ between pairs means $\Gamma^{i} \supset \Gamma^{i+1}$ and $A^{i} \supset A^{i+1}$, and where $\left(\Gamma^{i}, A^{i}\right)$ is a (possibly disconnected) relative metric ribbon graph for each $i=0, \ldots, d$. We say that $d$ is the depth of the filtration $\Gamma^{\bullet}$. We assume each $\Gamma^{i}$ does not have univalent vertices and is a subgraph of $\Gamma$ in the usual terminology in Graph Theory. We observe that since each $\left(\Gamma^{i}, A^{i}\right)$ is a relative metric ribbon graph, we have that $A^{i} \backslash A^{i+1}$ is a disjoint union of connected components homeomorphic to $\mathbb{S}^{1}$.

For each $i=0, \ldots, d$, let

$$
\delta_{i}: \Gamma^{i} \rightarrow \mathbb{R}_{\geq 0}
$$

be a locally constant map (so it is a map constant on each connected component). We put the restriction that $\delta_{0}\left(\Gamma^{0}\right)>0$. We denote the collection of all these maps by $\delta$.

Let $p \in \Gamma$, we define $c_{p}$ as the largest natural number such that $p \in \Gamma^{c_{p}}$.

Definition 5.1 (Mixed safe walk). Let $\left(\Gamma^{\bullet}, A^{\bullet}\right)$ be a filtered relative metric ribbon graph. Let $p \in \Gamma \backslash A \backslash v(\Gamma)$. We define a mixed safe walk $\gamma_{p}$ starting at $p$ as a concatenation of paths defined iteratively by the following properties 
i) $\gamma_{p}^{0}$ is a safe walk of length $\delta_{0}(p)$ starting at $p_{0}^{\gamma}:=p$. Let $p_{1}^{\gamma}:=\gamma^{0}\left(\delta_{0}\right)$ be its endpoint.

ii) Suppose that $\gamma_{p}^{i-1}$ is defined and let $p_{i}^{\gamma}$ be its endpoint.

- If $i>c_{p}$ or $p_{i}^{\gamma} \notin \Gamma^{i}$ we stop the algorithm.

- If $i \leq c_{p}$ and $p_{i}^{\gamma} \in \Gamma^{i}$ then define $\gamma_{p}^{i}:\left[0, \delta_{i}\left(p_{i}\right)\right] \rightarrow \Gamma^{i}$ to be a safe walk of length $\delta_{i}\left(p_{i}^{\gamma}\right)$ starting at $p_{i}^{\gamma}$ and going in the same direction as $\gamma_{p}^{i-1}$.

iii) Repeat step ii) until algorithm stops.

Finally, define $\gamma_{p}:=\gamma_{p}^{k} \star \cdots \star \gamma_{p}^{0}$, that is, the mixed safe walk starting at $p$ is the concatenation of all the safe walks defined in the inductive process above.

As in the pure case, there are two safe walks starting at each point on $\Gamma \backslash(A \cup$ $v(\Gamma))$. We denote them by $\gamma_{p}$ and $\omega_{p}$.

Definition 5.2 (Boundary mixed safe walk). Let $\left(\Gamma^{\bullet}, A^{\bullet}\right)$ be a filtered relative metric ribbon graph and let $p \in A$. We define a boundary mixed safe walk $b_{p}$ starting at $p$ as a concatenation of a collection of paths defined iteratively by the following properties

i) $b_{p_{0}}^{0}$ is a boundary safe walk of length $\delta_{0}(p)$ starting at $p_{0}:=p$ and going in the direction indicated by $A$ (as in the relative tête-à-tête case). Let $p_{1}:=b_{p}^{0}\left(\delta_{0}\right)$ be its endpoint.

ii) Suppose that $b_{p_{i-1}}^{i-1}$ is defined and let $p_{i}$ be its endpoint.

- If $i>c_{p}$ or $p_{i} \notin \Gamma^{i}$ we stop the algorithm.

- If $i \leq c(p)$ and $p_{i} \in \Gamma^{i}$ then define $b_{p_{i}}^{i}:\left[0, \delta_{i}\left(p_{i}\right)\right] \rightarrow \Gamma^{i}$ to be a safe walk of length $\delta_{i}\left(p_{i}\right)$ starting at $p_{i}$ and going in the same direction as $b_{p_{i-1}}^{i-1}$.

iii) Repeat step ii) until algorithm stops.

Finally, define $b_{p}:=b_{p_{k}}^{k} \star \cdots \star b_{p_{0}}^{0}$, that is, the boundary mixed safe walk starting at $p$ is the concatenation of all the safe walks defined in the inductive process.

Notation 5.3. We call the number $k$ in Definition 5.1 (resp. Definition 5.2), the order of the mixed safe walk (resp.boundary mixed safe walk) and denote it by $o\left(\gamma_{p}\right)\left(\right.$ resp. $\left.o\left(b_{p}\right)\right)$.

We denote by $l\left(\gamma_{p}\right)$ the length of the mixed safe walk $\gamma_{p}$ which is the sum $\sum_{j=0}^{o\left(\gamma_{p}\right)} \delta_{j}\left(p_{j}^{\gamma}\right)$ of the lengths of all the walks involved. We consider the analogous definition $l\left(b_{p}\right)$.

As in the pure case, two mixed safe walks starting at $p \in \Gamma \backslash v(\Gamma)$ exist. We denote by $\omega_{p}$ the mixed safe walk that starts at $p$ but in the opposite direction to the starting direction of $\gamma_{p}$.

Observe that since the safe walk $b_{p_{0}}^{0}$ is completely determined by $p$, for a point in $A$ there exists only one boundary safe walk.

Now we define the relative mixed tête-à-tête property.

Definition 5.4 (Relative mixed tête-à-tête property). Let $\left(\Gamma^{\bullet}, A^{\bullet}\right)$ be a filtered relative metric ribbon graph and let $\delta_{\bullet}$ be a set of locally constant mappings $\delta_{k}$ : $\Gamma^{k} \rightarrow \mathbb{R}_{\geq 0}$. We say that $\left(\Gamma^{\bullet}, A^{\bullet}, \delta_{\bullet}\right)$ satisfies the relative mixed tête-à-tête property or that it is a relative mixed tête-à-tête graph if for every $p \in \Gamma-(v(\Gamma) \cup A)$

I) The endpoints of $\gamma_{p}$ and $\omega_{p}$ coincide.

II) $c_{\gamma_{p}\left(l\left(\gamma_{p}\right)\right)}=c_{p}$ 
and for every $p \in A$, we have that

III) $b_{p}\left(l\left(b_{p}\right)\right) \in A^{c_{p}}$

As a consequence of the two previous definitions we have:

Lemma 5.5. Let $\left(\Gamma^{\bullet}, A^{\bullet}, \delta_{\bullet}\right)$ be a mixed relative tête-à-tête graph, then

a) $o\left(\omega_{p}\right)=o\left(\gamma_{p}\right)=c_{p}$

b) $l\left(\gamma_{p}\right)=l\left(\omega_{p}\right)$ for every $p \in \Gamma \backslash v(\Gamma)$.

Proof. See $[\mathrm{FPP}]$.

Remark 5.6. Note that for mixed tête-à-tête graphs it is not true that $p \mapsto$ $\gamma_{p}(\delta(p))$ gives a continuous mapping from $\Gamma$ to $\Gamma$.

Remark 5.7. Satisfying $I$ ) and $I I$ ) of the mixed tête-à-tête property in Definition 5.4 is equivalent to satisfying:

I') For all $i=0, \ldots, d-1$, the automorphism $\widetilde{\phi}_{\Gamma, i}=\mathcal{D}_{\delta_{i}} \circ \phi_{\Gamma, i-1}$ is compatible with the gluing $g_{i}$, that is,

$$
g_{i}(x)=g_{i}(y) \Rightarrow g_{i}\left(\widetilde{\phi}_{\Gamma, i}(x)\right)=g_{i}\left(\widetilde{\phi}_{\Gamma, i}(y)\right) .
$$

Below we see the diagram which shows the construction of $\phi_{\Gamma}$.

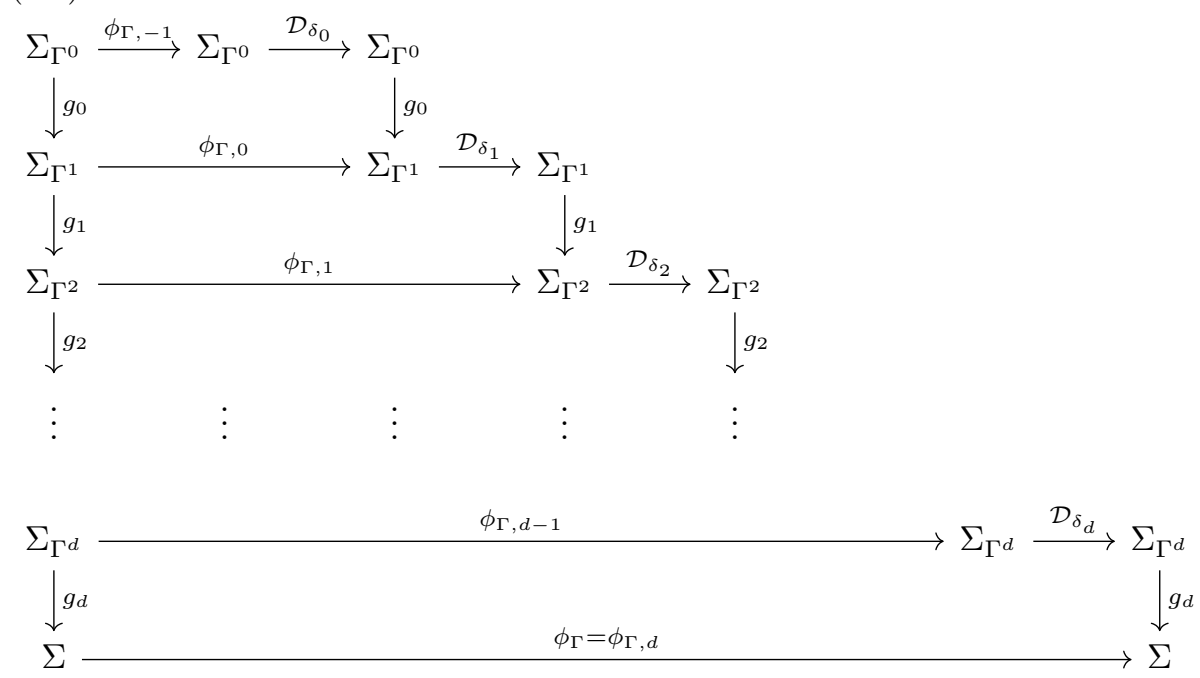

Remark 5.9. The pseudo-periodic automorphism $\phi_{\Gamma}$ induced by a mixed têteà-tête graph has negative screw numbers and positive fractional Dehn twist coefficients as noted in [FPP]. Actually, it was also proved in [FPP] that the screw number associated to an orbit of annuli $\mathcal{A}_{j, 1}^{i}, \ldots, \mathcal{A}_{j, k}^{i}$ between levels $i-1$ and $i$ of the filtration is

$$
-\sum_{k} \delta_{i}\left(\Gamma_{j, k}^{i}\right) / l\left(\tilde{\Gamma}_{j, 1}^{i}\right)
$$




\section{ReAlization THEOREM}

In this section we prove Theorem 6.7 which is the main results of this paper. It characterizes the pseudo-periodic automorphisms that can be realized by mixed tête-à-tête graphs. First we introduce some notation and conventions.

Let $\phi: \Sigma \rightarrow \Sigma$ be a pseudo-periodic automorphism. For the remaining of this work we impose the following restrictions on $\phi$ :

(i) The screw numbers are all negative.

(ii) It leaves at least one boundary component pointwise fixed and the fractional Dehn twist coefficients at these boundary components are positive.

Denote by $\partial^{1} \Sigma \subset \partial \Sigma$ the union of the boundary components pointwise fixed by $\phi$. We assume that $\phi$ is given in some almost-canonical form as in Remark 3.3.

Notation 6.1. We define a graph $G(\phi)$ associated to a given almost-canonical form:

(i) It has a vertex $v$ for each subsurface of $\Sigma \backslash \mathcal{A}$ whose connected components are cyclically permuted by $\phi$.

(ii) For each set of annuli in $\mathcal{A}$ permuted cyclically it has an edge connecting the vertices corresponding to the surfaces on each side of the collection of annuli.

Let $\mathcal{N}$ denote the set of vertices of $G(\phi)$.

Definition 6.2. We say that a function $L: \mathcal{N} \rightarrow \mathbb{Z}_{>0}$ is a filtering function for $G(\phi)$ if it satisfies:

(i) If $v, v^{\prime} \in \mathcal{N}$ are connected by an edge, then $L(v) \neq L\left(v^{\prime}\right)$.

(ii) If $v \in \mathcal{N}$, then either $v$ has a neighbor $v^{\prime} \in \mathcal{N}$ with $L(v)>L\left(v^{\prime}\right)$, or $L(v)=0$ and $\Sigma_{v}$ contains a component of $\partial^{1} \Sigma$.

Condition $i i$ ) above implies that for $L$ to be a filtering function, $L^{-1}(0)$ must only contain vertices corresponding to subsurfaces of $\Sigma \backslash \mathcal{A}$ that contain some component of $\partial^{1} \Sigma$. That same condition assures us that $L^{-1}(0)$ is non-empty.

Definition 6.3. Define the distance function $D: \mathcal{N} \rightarrow \mathbb{Z}_{\geq 0}$ as follows:

(i) $D(v)=0$ for all $v$ with $\Sigma_{v} \cap \partial^{1} \Sigma \neq \emptyset$.

(ii) $D(v)$ is the distance to the set $D^{-1}(0)$, that is the number of edges of the smallest bamboo in $G(\phi)$ connecting $v$ with some vertex in $D^{-1}(0)$.

Remark 6.4. Take some $\phi: \Sigma \rightarrow \Sigma$ in canonical form and observe that the function $D$ might not be a filtering function. It can happen that there are two adjacent vertices $v, v^{\prime} \in \mathcal{N}$ with $D(v)=D\left(v^{\prime}\right)$ or even that there is a vertex with a loop based at it (see Example 11.1). However we modify the canonical form into an almost-canonical form for which the function $D$ is a filtering function:

Let $\phi: \Sigma \rightarrow \Sigma$ be an automorphism in canonical form such that $D(v)=D\left(v^{\prime}\right)$ for some adjacent $v, v^{\prime} \in \mathcal{N}$. Take one edge joining $v$ and $v^{\prime}$, this edges corresponds to a set of annuli $\mathcal{A}_{1}, \ldots, \mathcal{A}_{k}$ being permuted cyclically by $\phi$. For each $i=1, \ldots, k$, let $\eta_{i}: \mathbb{S}^{1} \rightarrow \mathcal{A}_{i}$ be parametrizations as in Lemma 3.16. Let $\mathcal{C}_{i} \subset \mathcal{A}_{i}$ be the core curves of the annuli. We distinguish two cases:

1) The core curves are not amphidrome. By Remark 3.6 we can isotope $\phi$ on the annuli $\mathcal{A}_{i}$ to a automorphism $\tilde{\phi}$ without changing the action of $\phi$ on $\partial \mathcal{A}_{i}$ so that in the annuli $\eta_{i}\left(\mathbb{S}^{1} \times\left[\frac{1}{3}, \frac{2}{3}\right]\right)$ it is periodic. In doing so, we can redefine the canonical form to an almost-canonical form as follows. 
(a) for each $i=1, \ldots, k$ take $\mathcal{C}_{i}$ out from the set $\mathcal{C}$ and include $\eta_{i}\left(\mathbb{S}^{1} \times\left\{\frac{1}{6}\right\}\right)$ and $\eta_{i}\left(\mathbb{S}^{1} \times\left\{\frac{5}{6}\right\}\right)$.

(b) for each $i=1, \ldots, k$ take $\mathcal{A}_{i}$ out of $\mathcal{A}$ and include $\eta_{i}\left(\mathbb{S}^{1} \times[0,1 / 3]\right)$ and $\eta_{i}\left(\mathbb{S}^{1} \times\left[\frac{2}{3}, 1\right]\right)$.

It is clear that this new set of data defines an almost canonical form for $\tilde{\phi}$ and that on the corresponding $G(\tilde{\phi})$ the vertices $v$ and $v^{\prime}$ are no longer adjacent since a new vertex corresponding to the surface $\bigcup_{i} \eta_{i}\left(\mathbb{S}^{1} \times\left[\frac{1}{3}, \frac{2}{3}\right]\right)$ appears between them.

2) The core curves are amphidrome. This case is completely analogous to case 1) with the advantage that by definition of $\tilde{\mathcal{D}}_{s}$ inNotation 3.8 , it is already periodic in the central annuli.

It is clear that after performing 1) or 2) (accordingly) for all pairs of adjacent vertices $v, v^{\prime}$ with $D(v)=D\left(v^{\prime}\right)$ we provide $\phi$ with an almost-canonical whose distance function $D$ is a filtering function.

Remark 6.5. We observe that orbits of amphidrome annuli $\mathcal{A}_{1}, \ldots, \mathcal{A}_{i}$ correspond to loops in $G(\phi)$. So we have that after performing the modification of Remark 6.4, the almost-canonical form of $\phi$ does not have any amphidrome annuli in $\mathcal{A}$. However, some of the surfaces of $\Sigma \backslash \mathcal{A}$ are now amphidrome annuli.

Notation 6.6. We assume $\phi$ is in the almost-canonical form induced from the canonical form after performing the modification described in Remark 6.4. We denote by $\hat{\Sigma}$ the closure of $\Sigma \backslash \mathcal{A}$ in $\Sigma$. Let $\hat{G}(\phi)$ be a graph constructed as follows:

(i) It has a vertex for each connected component of $\Sigma \backslash \mathcal{C}$.

(ii) There are as many edges joining two vertices as curves in $\mathcal{C}$ intersect the two surfaces corresponding to those vertices.

We observe that the previously defined $G(\phi)$ is nothing but the quotient of $\hat{G}(\phi)$ by the action induced by $\phi$ on the connected components of $\Sigma \backslash \mathcal{A}$.

Let $\hat{\mathcal{N}}$ be the set of vertices of $\hat{G}(\phi)$. Since $\phi$ permutes the surfaces in $\hat{\Sigma}$, it induces a permutation of the set $\hat{\mathcal{N}}$ which we denote by $\sigma_{\phi}$. We label the set $\hat{\mathcal{N}}$, as well as the connected components of $\hat{\Sigma}$ and the connected components of $\mathcal{A}$ in the following way:

(i) Label the vertices that correspond to surfaces containing components of $\partial^{1} \Sigma$ by $v_{1,1}^{0}, v_{2,1}^{0}, \ldots, v_{\beta_{0}, 1}^{0}$. Let $V^{0}$ be the union of these vertices. Note that $\sigma_{\phi}\left(v_{j, 1}^{0}\right)=v_{j, 1}^{0}$ for all $j=1, \ldots, \beta_{0}$.

(ii) Let $\hat{D}: \hat{\mathcal{N}} \rightarrow \mathbb{Z}_{\geq 0}$ be the distance function to $V^{0}$, that is, $\hat{D}(v)$ is the number of edges of the smallest path in $\hat{G}(\phi)$ that joins $v$ with $V^{0}$. Let $V^{i}:=\hat{D}^{-1}(i)$. Observe that the permutation $\sigma_{\phi}$ leaves the set $V^{i}$ invariant. There is a labeling of $V^{i}$ induced by the orbits of $\sigma_{\phi}$ : suppose it has $\beta_{i}$ different orbits. For each $j=1, \ldots, \beta_{i}$, we label the vertices in that orbit by $v_{j, k}^{i}$ with $k=1, \ldots, \alpha_{j}$ so that $\sigma_{\phi}\left(v_{j, k}^{i}\right)=v_{j, k+1}^{i}$ and $\sigma_{\phi}\left(v_{j, \alpha_{j}}^{i}\right)=v_{j, 1}^{i}$.

Denote by $\Sigma_{j, k}^{i}$ the surface in $\hat{\Sigma}$ corresponding to the vertex $v_{j, k}^{i}$. Denote by $\Sigma^{i}$ the union of the surfaces corresponding to the vertices in $V^{i}$. We denote by $\Sigma \leq i$ the union of $\Sigma^{0}, \ldots, \Sigma^{i}$ and the annuli in between them.

We recall that $\alpha_{j}$ is the smallest positive number such that $\phi^{\alpha_{j}}\left(\Sigma_{j, k}^{i}\right)=\Sigma_{j, k}^{i}$. 
Theorem 6.7. Let $\phi: \Sigma \rightarrow \Sigma$ be a pseudo-periodic automorphism satisfying assumptions (i) and (ii). Then there exists a relative mixed tête-à-tête graph $\left(\Gamma^{\bullet}, A^{\bullet}, \delta_{\bullet}\right)$ with $\Gamma$ embedded in $\Sigma$ such that:

(i) $\delta_{i}$ is a constant function for each $i=1, \ldots, d$.

(ii) $[\phi]_{\partial^{1} \Sigma}=\left[\phi_{\Gamma}\right]_{\partial^{1} \Sigma}$.

(iii) $\left.\phi\right|_{\partial \Sigma \backslash \partial^{1} \Sigma}=\left.\phi_{\Gamma}\right|_{\partial \Sigma \backslash \partial^{1} \Sigma}$.

(iv) Filtration indexes are induced by the distance function $D$ for the almostcanonical form induced from the canonical form by Remark 6.4.

Now we state and prove Lemma 6.8 and Lemma 6.9 which are used in the proof of Theorem 6.7 .

Lemma 6.8. Let $\phi: \Sigma \rightarrow \Sigma$ be a periodic automorphism of order $n$. Let $C=$ $C_{1} \sqcup \cdots \sqcup C_{k}$ be a non-empty collection of boundary components of $\Sigma$ such that $\phi\left(C_{i}\right)=C_{i}$, that is, each one is invariant by $\phi$. For each $i$ let $m_{i}$ be a metric on $C_{i}$ invariant by $\phi$. Then there exists a relative metric ribbon graph $(\Gamma, A) \hookrightarrow(\Sigma, \partial \Sigma \backslash C)$ and parametrizations of the cylinders (see Notation 4.4) $r_{i}: \mathbb{S}^{1} \times I \rightarrow \tilde{\Sigma}_{i}$ such that:

(i) $\phi(\Gamma)=\Gamma$ and the metric of $\Gamma$ is also invariant by $\phi$.

(ii) $l\left(\tilde{\Gamma}_{i}\right)=l\left(C_{i}\right)$.

(iii) The projection from $C_{i}$ to $\tilde{\Gamma}_{i}$ induced by $r_{i}$ is an isometry, that is, the map

$$
r(\theta, 0) \mapsto r(\theta, 1)
$$

is an isometry.

(iv) $\phi$ sends retractions lines (i.e. $\{\theta\} \times I)$ to retractions lines.

Proof. The proof uses essentially the same technique used in the proof of Theorem 5.4 and 6.2 in [FPP]. For completeness we outline it here.

Let $\Sigma^{\phi}$ be the orbit surface and suppose it has genus $g$ and $r \geq k$ boundary components. Let $p: \Sigma \rightarrow \Sigma^{\phi}$ be the induced branch cover.

Take any relative spine $\Gamma^{\phi}$ of $\Sigma^{\phi}$ that:

1) Contains all branch points of the map $p$.

2) Contains the boundary components $p(\partial \Sigma \backslash C)$.

3) Admits a metric such that $p\left(C_{i}\right)$ retracts to a part of the graph of length $l\left(C_{i}\right) / n$.

We observe that conditions 1) are 2) are trivial to get. Condition 3) follows because of the proof of Theorem 5.4 and 6.2 in [FPP]. There, the conditions on the metric of the graph $\Gamma^{\phi}$ come from the rotation numbers of $\phi$, however, we do not use that these numbers come from $\phi$ in finding the appropriate graph so exactly the same argument applies.

Observe that since the metric on $C_{i}$ is invariant by $\phi$, there is a metric induced on $p\left(C_{i}\right)$ for $i=1, \ldots k$. Now choose any parametrizations (or product structures) of the cylinders in $\Sigma_{\Gamma^{\phi}}^{\phi}$ such that their retractions lines induce an isometry from $p\left(C_{i}\right)$ to $\tilde{\Gamma}_{i}^{\phi}$.

Define $\Gamma:=p^{-1}\left(\Gamma^{\phi}\right)$. By construction, this graph satisfies $(i)$ and $(i i)$. The preimage by $p$ of retraction lines on $\Sigma^{\phi}$ gives rise to parametrizations of the cylinders in $\Sigma_{\Gamma}$ satisfying $\left.i i i\right)$ and $i v$ ).

Lemma 6.9. Let $\left(\Gamma^{\bullet}, B^{\bullet}, \delta_{\bullet}\right)$ be a relative mixed tête-à-tête graph embedded in a surface $\Sigma$ and let $C_{1}, \ldots, C_{k} \subset B$ a set of relative boundary components cyclically permuted by $\phi$. Suppose that all the vertices in these boundary components are of valency 3. Then we can modify the metric structure of the graph to produce 
a mixed tête-à-tête graph $\left(\hat{\Gamma}^{\bullet}, \hat{B}^{\bullet}, \delta_{\bullet}\right)$ with $l\left(C_{i}\right)$ as small as we want and with $\left[\phi_{\Gamma}\right]_{\partial^{1} \Sigma}=\left[\phi_{\hat{\Gamma}}\right]_{\partial^{1} \Sigma}$.

Proof. Let $e_{1}, \ldots, e_{m}$ be the edges comprising $C_{1}$, where $e_{j}$ has length $l_{j}$. Let $v_{1}, \ldots, v_{m}$ be the vertices of these edges, so that $e_{i}$ connects $v_{i}$ and $v_{i+1}$ (here, indices are taken modulo $m$ ). Let $f_{i, j}$, for $j=1, \ldots, n_{i}$ be the edges adjacent to $v_{i}$, other than $e_{i}, e_{i+1}$, in such a way that the edges have the cyclic order $e_{i+1}, e_{i}, f_{i, 1}, \ldots, f_{i, n_{i}}$. Let $\varepsilon<l\left(C_{1}\right)$. We would like to replace $C_{1}$ with a circle of length $l\left(C_{1}\right)-\varepsilon$. We assume that $l_{1}=\min _{i} l_{i}$.

If $\varepsilon / m \leq l_{1}$, then we do the following:

Each edge $e_{i}$ is modified to have length $l_{i}-\varepsilon / m$.

For any $i$ with $n_{i}=1$, the length of $f_{i, 1}$ is increased by $\varepsilon / 2 m$.

For any $i$ with $n_{i}>1$, extrude an edge $g_{i}$ from the vertex $v_{i}$ of length $\varepsilon / 2 m$ so that one end of $g_{i}$ is adjacent to $e_{i}, e_{i+1}$ and $g_{i}$, and the other is adjacent to $f_{i, 1}, \ldots, f_{i, n_{i}}$ and $g_{i}$, with these cyclic orders.

In the case when $\varepsilon / m>l_{1}$, we execute the above procedure with $\varepsilon$ replaced by $m \cdot l_{1}$, which results in a circle made up of fewer edges. After finitely many steps, we obtain the desired length for $C_{1}$.

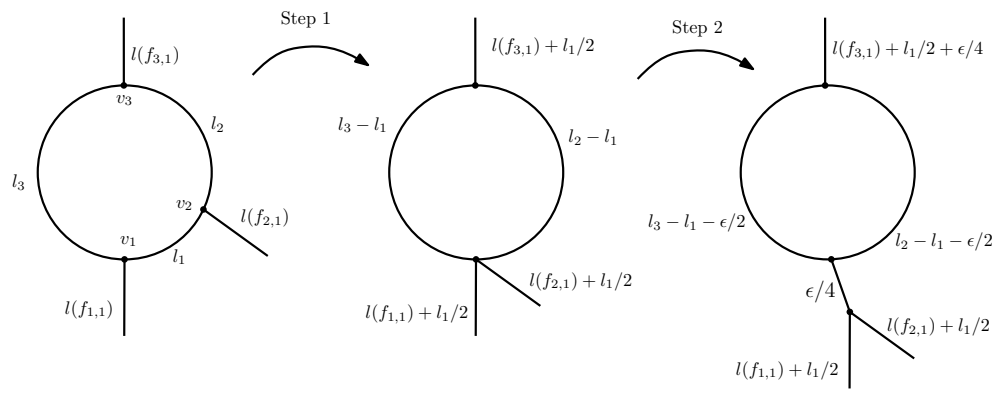

Figure 6.1. Example of modification at a boundary component. Suppose that $l_{1}<l_{2}<l_{3}$. In step 1 we reduce the length of the circle by $l_{1}$. In step 2 we reduce it by $\varepsilon$.

Proof of Theorem 6.7. By definition, all surfaces corresponding to vertices in $D^{-1}(0)$ are connected because they are invariant by $\phi$. We have that $\left.\phi\right|_{\Sigma_{j, 1}^{0}}: \Sigma_{j, 1}^{0} \rightarrow \Sigma_{j, 1}^{0}$ is periodic outside a neighborhood of $\partial^{1} \Sigma \cap \Sigma_{j, 1}^{0}$ and that the fractional Dehn twist coefficients with respect to all the components in $\partial^{1} \Sigma \cap \Sigma_{j, 1}^{0}$ are positive. Denote $B_{j, 1}^{0}:=\partial \Sigma_{j, 1}^{0} \backslash \partial^{1} \Sigma$. By Theorem 4.6, for each $j=1, \ldots, \beta_{0}$, there is a relative têteà-tête graph $\left(\Gamma_{j, 1}^{0}, B_{j, 1}^{0}\right)$ embedded in $\Sigma_{j, 1}^{0}$ modeling $\left.\phi\right|_{\Gamma_{j, 1}^{0}}$. Denote $\Gamma[0]:=\bigsqcup_{j} \Gamma_{j, 1}^{0}$ and $B[0]:=\bigsqcup_{j} B_{j, 1}^{0}$. Then $\left(\Gamma[0], B[0], \delta_{0}\right)$ is a relative mixed tête-à-tête graph of depth 0 for $\Sigma^{\leq 0}$ (it is just a relative tête-à-tête graph) such that:

(i) $\left.\delta_{0}\right|_{\Sigma_{j, 1}^{0}}=\pi$ for all $j=1, \ldots, \beta_{0}$.

(ii) $\left[\left.\phi\right|_{\Sigma^{0}}\right]_{\partial^{1} \Sigma^{0}}=\left[\phi_{\Gamma[0]}\right]_{\partial^{1} \Sigma^{0}}$.

(iii) $\left.\phi\right|_{B[0]}=\left.\phi_{\Gamma[0]}\right|_{B[0]}$.

(iv) All the vertices on $B[0]$ have valency 3 . 
Suppose that we have a relative mixed tête-à-tête graph $\left(\Gamma[a-1]^{\bullet}, B[a-1]^{\bullet}, \delta[a-\right.$ 1]•) of depth $a$ embedded as a spine in $\Sigma^{\leq a}$ and with $B[a-1]=\partial \Sigma \leq a-1 \backslash \partial^{1} \Sigma$ such that:

(i) $\delta[a-1]_{i}$ is a constant function for each $i=0, \ldots, a-1$

(ii) $\left[\left.\phi\right|_{\Sigma \leq a-1}\right]_{\partial^{1} \Sigma \leq a-1}=\left[\phi_{\Gamma[a-1]}\right]_{\partial^{1} \Sigma \leq a-1}$

(iii) $\left.\phi\right|_{B[a-1]}=\left.\phi_{\Gamma[a-1]}\right|_{B[a-1]}$.

(iv) All the vertices on $B[a-1]$ have valency 3 .

We recall that $\phi_{\Gamma[a-1]}$ denotes the mixed tête-à-tête automorphism induced by $\left(\Gamma[a-1]^{\bullet}, B[a-1]^{\bullet}, \delta[a-1]_{\bullet}\right)$. We extend $\Gamma[a-1]$ to a mixed tête-à-tête graph $\Gamma[a]$ satisfying (i) - (iv). This proves the theorem by induction. We focus on a particular orbit of surfaces. Fix $j \in\left\{1, \ldots, \beta_{a}\right\}$ and consider the surfaces $\Sigma_{j, 1}^{a}, \ldots, \Sigma_{j, \alpha_{j}}^{a} \subset \Sigma^{a}$ with $\phi\left(\Sigma_{j, k}^{a}\right)=\Sigma_{j, k+1}^{a}$ and $\phi\left(\Sigma_{j, \alpha_{j}}^{a}\right)=\Sigma_{j, 1}^{a}$.

For each $j$, we distinguish two types of boundary components in the orbit $\bigsqcup_{k} \Sigma_{j, k}^{a}$ :

Type I) Boundary components that are connected to an annulus whose other end is in $\Sigma^{a-1}$, we denote these by $\partial^{I}$.

Type II) The rest: boundary components that are in $\partial \Sigma$ and boundary components that are connected to an annulus whose other end is in $\Sigma^{a+1}$, we denote these by $\partial^{I I}$.

Since we are doing the construction for an orbit, we use local notation in which not all the indices are specified so that the formulae is easier to read.

Let $\mathcal{A}^{I}$ denote the union of annuli connected to boundary components in $\partial^{I}$. These annuli are permuted by $\phi$. Suppose that there are $r^{\prime}$ different orbits of annuli $\mathcal{A}_{1}, \ldots \mathcal{A}_{r^{\prime}}$, and let $\ell_{i} \in \mathbb{N}$ be the length of the orbit $\mathcal{A}_{i}$. Let $s_{i}$ be the screw number of the orbit $\mathcal{A}_{i}$ (recall Definition 3.14 and Remark 3.15 ). Let $\mathcal{B}_{i, 1}, \ldots, \mathcal{B}_{i, \ell_{i}}$ be the orbit of boundary components of $\Sigma^{a-1}$ that are contained in the orbit $\mathcal{A}_{i}$. The metric of $\Gamma[a-1]$ gives lengths to these boundary components and all the boundary components in the same orbit have the same length $l\left(\mathcal{B}_{i, 1}\right) \in \mathbb{R}_{+}$. Consider the positive real numbers

$$
\frac{s_{1}}{\ell_{1}} l\left(\mathcal{B}_{1,1}\right), \ldots, \frac{s_{r^{\prime}}}{\ell_{r^{\prime}}} l\left(\mathcal{B}_{r^{\prime}, 1}\right)
$$

Using Lemma 6.9 , we modify the metric structure of $\Gamma[a-1]$ near each orbit $\mathcal{B}_{i}$ so that

$$
\frac{s_{1}}{\ell_{1}} l\left(\mathcal{B}_{1,1}\right)=\cdots=\frac{s_{r^{\prime}}}{\ell_{r^{\prime}}} l\left(\mathcal{B}_{r^{\prime}, 1}\right) .
$$

This is possible since we can make $l\left(\mathcal{B}_{i, 1}\right)$ as small as needed.

For each $i=1, \ldots, r^{\prime}$, let $\mathcal{A}_{i, 1}, \ldots \mathcal{A}_{i, \ell_{i}}$ be the annuli in the orbit $\mathcal{A}_{i}$ and let $\mathcal{B}_{i, 1}^{\prime}, \ldots \mathcal{B}_{i, \ell_{i}}^{\prime}$ be the boundary components that they share with $\Sigma^{a}$. Consider parametrizations $\eta_{i, 1}, \ldots, \eta_{\ell_{i}, 1}$ given by Lemma 3.16. The metric on the boundary components of $B[a-1]$ and the parametrizations induce a metric on all the boundary components in $\partial^{I}$ that is invariant by $\phi$.

We observe that $\left.\phi^{\alpha_{j}}\right|_{\Sigma_{j, 1}^{a}}: \Sigma_{j, 1}^{a} \rightarrow \Sigma_{j, 1}^{a}$ is periodic and $\partial^{I} \cap \Sigma_{j, 1}^{a}$ is a subset of boundary components that have a metric. So we can apply Lemma 6.8 and we get a relative metric ribbon graph $\left(\Gamma_{j, 1}^{a}, \partial^{I I} \cap \Gamma_{j, 1}^{a}\right)$ and parametrizations of each cylinder in $\left(\Sigma_{j, 1}^{a}\right)_{\Gamma_{j, 1}^{a}}$ with properties $\left.\left.i\right), \ldots, i v\right)$ in the the Lemma. We can translate this construction by $\phi$ to the rest of the surfaces $\Sigma_{j, 2}^{a}, \ldots, \Sigma_{j, \alpha_{j}}^{a}$. So we get graphs $\Gamma_{j, k}^{a} \hookrightarrow \Sigma_{j, k}^{a}$ and parametrizations for the cylinders in $\left(\Sigma_{j, k}^{a}\right)_{\Gamma_{j, k}^{a}}$ for all 
$k=1, \ldots, \alpha_{j}$. The construction assures us that $\left.\phi\right|_{\Sigma_{j, \alpha_{j}}^{a}}: \Sigma_{j, \alpha_{j}}^{a} \rightarrow \Sigma_{j, 1}^{a}$ sends $\Gamma_{j, \alpha_{j}}^{a}$ to $\Gamma_{j, 1}^{a}$ isometrically and that it takes retractions lines of the parametrizations in $\Sigma_{j, \alpha_{j}}^{a}$ to retraction lines in $\Sigma_{j, 1}^{a}$.

We proceed to extend $\Gamma[a-1]$ to the orbit of $\Sigma_{j, 1}^{a}$. For each $i=1, \ldots, r^{\prime}$ do the following:

Step 1. Remove $\mathcal{B}_{i, 1}, \ldots, \mathcal{B}_{i, \ell_{i}}$ from $\Gamma[a-1]$.

Step 2. Take $\varepsilon>0$ small enough. Decrease by $\varepsilon$ the metric on all the edges of $\Gamma[a-1]$ adjacent to vertices in $\mathcal{B}_{i, 1}, \ldots, \mathcal{B}_{i, \ell_{i}}$.

Step 3. Add to the graph the retraction lines of the parametrizations $\eta_{i, 1}, \ldots, \eta_{i, \ell_{i}}$ that were adjacent to vertices in $\mathcal{B}_{i, 1}, \ldots, \mathcal{B}_{i, \ell_{i}}$. That is, if $v \in \mathcal{B}_{i, 1} \subset \mathcal{A}_{i, 1}$ include $\eta_{i, 1}(\{v\} \times I)$. Define the length of these segments as $\varepsilon / 2$.

Step 4. Add to the graph the retraction lines of the parametrizations of the cylinders $\left(\Sigma_{j, k}^{a}\right)_{\Gamma_{j, k}^{a}}^{a}$ that start at the ends of the lines added in the previous step. Define the length of these segments as $\varepsilon / 2$.

Step 5. Add to the graph the graphs $\Gamma_{j, 1}^{a}, \ldots, \Gamma_{j, \alpha_{j}}^{a}$.

We repeat this process for all orbits of surfaces in $\Sigma^{a}$ and so we extend the graph $\Gamma[a-1]$ to all $\Sigma^{a}$. Denote

$$
\Gamma[a]^{a}:=\bigsqcup_{j, k} \Gamma_{j, k}^{a} .
$$

Denote the resulting graph by $\Gamma[a]$.

We make the following observation: $\left(\Gamma[a]_{\Gamma[a]^{a}}, \tilde{\Gamma}[a]^{a}\right)$ is by construction isometric to $(\Gamma[a-1], \mathcal{B}[a-1])$. We denote the induced relative mixed tête-à-tête automorphism by $\phi_{\Gamma[a]_{\Gamma[a]}^{a}}$ which acts on $\Sigma_{\Gamma[a]^{a}}^{\leq a}$. By the previous observation there is an induced filtration on $\Gamma[a]$ :

$$
\Gamma[a]=\Gamma[a]^{0} \supset \Gamma[a]^{1} \supset \cdots \supset \Gamma[a]^{a-1} \supset \Gamma[a]^{a}
$$

and similarly for the relative parts. We define $\delta_{a}: \Gamma[a]^{a} \rightarrow \mathbb{R}_{\geq} 0$ to be the constant function equal to the numbers 6.10 (which are by construction the same number).

By the choice of $\delta_{a}$ and the parametrizations on the annuli that join $\Sigma^{a-1}$ with $\Sigma^{a}$ we have that

$$
\mathcal{D}_{\delta_{a}} \circ \phi_{\Gamma_{\Gamma[a]}}: \Sigma_{\Gamma[a]^{a}} \rightarrow \Sigma_{\Gamma[a]^{a}}
$$

is compatible with the gluing $g_{a+1}$. So that $(\Gamma[a], \mathcal{B}[a])$ is a relative mixed tête-àtête graph follows from $I^{\prime}$ ) in Remark 5.7.

We have already made sure in the construction that (i) and (iv) hold in $\Gamma[a]$.

Let's show that (ii) and (iii) also hold. Observe that by construction $\phi$ leaves $\Gamma[a]$ invariant so there is an automorphism $\tilde{\phi}_{a}: \Sigma_{\Gamma[a]^{a}} \rightarrow \Sigma_{\Gamma[a]^{a}}$ induced. This automorphism coincides with $\mathcal{D}_{\delta_{a}} \circ \phi_{\Gamma_{\Gamma[a]}}$ on $\tilde{\Gamma}[a]$ by the choice of the parametrizations of the annuli $\mathcal{A}$ and by the choice of the number $\delta_{a}$. Also, by the choice of $\delta_{a}$ and [FPP, Remark 8.12] we see that they have the same screw numbers on the annuli connecting the level $a-1$ and the level $a$. From this discussion we get (ii) and (iii) and finish the proof.

Remark 6.11. From the proof we get as an important consequence that a more restrictive definition of a mixed tête-à-tête graph is valid: it is enough to consider mixed tête-à-tête graphs where $\delta_{i}$ is a constant function (i.e. a number) for all $i=0, \ldots, \ell$. 
Corollary 6.12. The monodromy associated with a reduced holomorphic function germ defined on an isolated surface singularity is a mixed tête-à-tête twist. Conversely, let $C(\Gamma)$ be the cone over the open book associated with a mixed tête-à-tête graph. Then there exists a complex structure on $C(\Gamma)$ and a reduced holomorphic function germ $f: C(\Gamma) \rightarrow \mathbb{C}$ inducing $\phi_{\Gamma}$ as the monodromy of its Milnor fibration.

Proof. The plumbing graph associated to a reduced holomorphic function germ has positive signs at all edges and induces positive multiplicity weights. Then by Lemma 8.2, we find that this monodromy has negative screw numbers and positive fractional Dehn twist coefficients. Therefore, we conclude the first part of the statement from Theorem 6.7.

The second part of the statement follows from Theorem 6.7 and [NP07, Theorem 2.1].

\section{HORIZONTAL OPEN BOOKS IN GRAPH MANIFOLDS}

In this section we review some theory about graph manifolds and their fibrations over the circle. The section is divided in two parts. In the first part we fix notation and review some results on Waldhausen and plumbing graphs. In the second part we state and prove results on horizontal fibrations of Waldhausen links.

The main result in this part is Proposition 7.10 which classifies horizontal fibrations of link complements by those cohomology classes which do not vanish on generic fibers in Seifert pieces. On one hand, this statement is proved in [EN85] when $Y$ is an integral homology sphere. It is proved there as well that a homotopy class in $\left[Y, S^{1}\right]$ contains at most one fibration. A criterion is also given in [Neu97] for a graph manifold to fiber over $S^{1}$ and have a horizontal surface. On the other hand, the results of [Pic01] provide existence of fibrations of a link complement $Y \backslash L$, by constructing $Y \backslash L$ as the mapping torus of a surface automorphism. For the sake of completeness, we state and prove this result which helps us clarify the setting used in Sections 9 and 10.

Let $Y$ be a graph manifold, and let $(Y, L)$ be a Waldhausen link (see e.g. [Pic01] pg. 338). We assume that $Y$ is oriented and that the fibers of each Seifert piece are also oriented, so this gives an orientation to each connected component $L_{k}$ of $L$.

Definition 7.1. We say that $L$ is a fibered link if there exists a locally trivial fibration $f: Y \backslash L \rightarrow \mathbb{S}^{1}$ and for each connected component $L_{k} \subset L$ there exists a tubular neighborhood $U_{k}$ and a trivialization $\rho_{k}: \bar{U}_{k} \rightarrow D^{2} \times \mathbb{S}^{1}$ such that the following diagram commutes

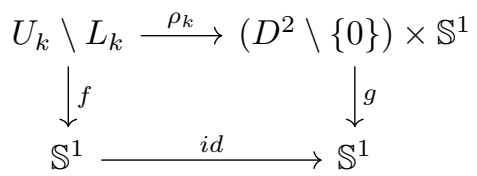

where $g(z, t)=\left(\frac{z}{|z|}\right)^{m_{k}} \cdot t^{n_{k}}$.

We call $m_{k}$ the multiplicity of the component $L_{k}$, it coincides with the intersection number of a fiber $f^{-1}(t)$ and a meridian $\partial \rho_{k}^{-1}\left(D^{2} \times\{t\}\right)$ around $L_{k}$. When $m_{k}=+1$ for all the components in $L$, we call $f$ an open book decomposition of $Y$. We note that $n_{k}$ is determined modulo $m_{k}$. 
If the fibers $f^{-1}(t)$ are transverse to the Seifert fibers of each Seifert piece of $Y \backslash L$, then we say that it is a horizontal fibration for the pair $(Y, L)$. And when $m_{k}=+1$ for all the components in $L$ we say that it is a horizontal open book.

Waldhausen and plumbing graphs. We start by introducing Waldhausen and plumbing graphs. For more on this topic, see [Wal67, Neu81].

7.3. Let $Y$ be a closed oriented graph manifold and let $L \subset Y$ be a Waldhausen-link. Thus, we have $Y=\cup_{n \in \mathcal{N}} Y_{n}$, where each $Y_{n}$ is a Seifert manifold. Decompose $L$ into connected components as $L=\cup_{a \in \mathcal{A}} L_{a}$. For each $a \in \mathcal{A}$, we have an $n(a) \in \mathcal{N}$ so that $L_{a} \subset Y_{n(a)}$ is a Seifert fiber.

Let $G(Y, L)$ be a graph defined as follows. It has vertex set $\mathcal{N} \cup \mathcal{A}$, where the vertices $a \in \mathcal{A}$ are arrow-heads. For each $n \in \mathcal{N}$, let $S_{n}$ be a general fiber in $Y_{n}$, and let $S_{n, 1}, \ldots, S_{n, k_{n}}$ be the special fibers. Furthermore, for $a \in \mathcal{A}$, let $S_{a}$ be a small meridian around $L_{a}$.

An edge $e$ connecting the vertices $n, n^{\prime} \in \mathcal{N}$ corresponds to a torus $T_{e} \subset Y_{n} \cap Y_{n^{\prime}}$. Assuming that we have chosen $S_{n}, S_{n^{\prime}} \subset T_{e}$, orient $T_{e}$ as part of $\partial Y_{n}$ and define

$$
\alpha_{e}=\left|\left(S_{n}, S_{n^{\prime}}\right)_{T_{e}}\right|, \quad \varepsilon_{e}=\operatorname{sign}\left(S_{n}, S_{n^{\prime}}\right)_{T_{e}} .
$$

Taking $e$ as an oriented edge going from $n$ to $n^{\prime}$, we define $\beta_{e}$ as the unique integer satisfying $0 \leq \beta_{e}<\alpha_{e}$ and $\beta_{e} S_{n}+S_{n^{\prime}} \in \alpha_{e} \cdot H_{1}\left(T_{e}, \mathbb{Z}\right)$. Note that if we denote by $e^{\prime}$ the edge $e$ with the opposite orientation, then we have $\beta_{e} \beta_{e^{\prime}} \equiv 1\left(\bmod \alpha_{e}\right)$. If $e$ is an edge connecting $n(a)$ and $a \in \mathcal{A}$, then $\alpha_{e}$ and $\beta_{e}$ are defined as the Seifert invariants associated with the fiber $L_{a} \subset Y_{n(a)}$.

Let $\Sigma_{n}$ be a horizontal surface in $Y_{n} \backslash L$. Such a surface exists, unless $Y_{n}$ is a closed Seifert manifold, $L=\emptyset$ and the orbifold Euler characteristic is nonzero. The intersection number between $S_{n}$ and $\Sigma_{n}$ is independent of the general Seifert fiber $S_{n}$, call this number $m_{n}$. With a correct orientation for $\Sigma_{n}$, we can assume that $m_{n}>0$. Orient $\Sigma_{n} \cap T_{e}$ as the boundary of $\Sigma_{n}$. Orient any edge adjacent to $n$ as going out of $n$. We find $\left(\Sigma_{n} \cap T_{e}, S_{n}\right)_{T_{e}}=m_{n}$.

Observe that $S_{n}, S_{n^{\prime}}$ is a basis of $H_{1}\left(T_{e} ; \mathbb{Q}\right)$. We find that $\Sigma_{n} \cap T_{e}$ is the sum of $-m_{n} \varepsilon_{e} S_{n^{\prime}} / \alpha_{e}$ and some rational multiple of $S_{n}$. Let $N_{L}$ be an open tubular neighborhood around $L$ in $Y$. Since $\partial\left(\Sigma_{n} \backslash N_{L}\right)=\cup_{e} \Sigma_{n} \cap T_{e}$, there exists a number $e_{n} \in \mathbb{Q}$ so that

$$
e_{n} S_{n}+\sum_{e} \frac{\varepsilon_{e}}{\alpha_{e}} S_{n^{\prime}}=0 \in H_{1}\left(Y_{n}, \mathbb{Z}\right)
$$

where $e$ ranges over oriented edges going from $n$ to any $n^{\prime}$. Since $m_{n} \neq 0$, the fiber $S_{n}$ does not represent a torsion element of $H_{1}\left(Y_{n}, \mathbb{Z}\right)$, and so $e_{n}$ is well defined by this equation. We call $e_{n}$ the orbifold Euler number associated with $n$. We define the orbifold intersection matrix $I^{\mathrm{O}}$ by setting

$$
I_{n, n}^{\mathrm{o}}=e_{n}, \quad I_{n, n^{\prime}}^{\mathrm{o}}=\sum_{e} 1 / \alpha_{e} \quad n \neq n^{\prime},
$$

where the sum runs over edges connecting $n$ and $n^{\prime}$.

7.5. We can replace the above data with a plumbing graph $\Lambda$. Any vertex $v$ in $\Lambda$ is decorated with an Euler number $-b_{v}$ and a genus $g_{v}$. It is obtained from $G(Y, L)$ as follows. Replace any edge $e$ connecting $n, n^{\prime} \in \mathcal{N}$ by a string of vertices with genus zero and Euler numbers $-b_{1}, \ldots,-b_{s}$, the sequence given by the negative 
continued fraction expansion

$$
\left[b_{1}, \ldots, b_{s}\right]= \begin{cases}\frac{\alpha_{e}}{\beta_{e}} & \varepsilon_{e}=+1, \\ \frac{\alpha_{e}}{\alpha_{e}-\beta_{e}} & \varepsilon_{e}=-1 .\end{cases}
$$

If $\varepsilon_{e}=+1$, then the edges in this bamboo are positive. If $\varepsilon_{e}=-1$, then one (or any odd number) of the edges are given a negative sign. The vertex $n$ has genus decoration given by the genus of the base space of the corresponding Seifert fibration and the Euler number associated with $n$ is obtained from the formula

$$
e_{n}=-b_{n}+\sum_{e} \beta_{e} / \alpha_{e}
$$

where the sum ranges over all (oriented) edges starting at $n$. We note that a plumbing graph is the same as a Waldhausen graph in the case when all $\alpha_{e}$ are 1 and all Seifert fibrations have no special fibers. Also, the set of non-arrowhead vertices $\mathcal{N}$ in $G(Y, L)$ is naturally seen as a subset of the set of non-arrowhead vertices $\mathcal{V}$ of $\Lambda$. Furthermore, they share the same set of arrowhead vertices $\mathcal{A}$. Corresponding to eq. (7.4), we find for any $v \in \mathcal{V}$

$$
-b_{v} S_{v}+\sum_{e} \varepsilon_{e} S_{w}=0 \in H_{1}\left(Y_{n}, \mathbb{Z}\right)
$$

where the sum ranges over edges adjacent to $v$, and $w$ is the other vertex adjacent to that edge. In the case of a plumbing graph, the orbifold intersection matrix is simply called the intersection matrix and is denoted by $I$. This is an integral matrix.

7.8. In the setting of pseudo-periodic automorphisms arising from mixed tête-àtête graphs, we can safely assume that $\varepsilon_{e}=+1$ for every edge in the plumbing graph. By assuming that both the multiplicities $m_{n}$ and the signs $\varepsilon_{e}$ are positive, we are only considering horizontal surfaces whose monodromy has only positive fractional Dehn twist coefficients and negative screw numbers. This is derived from computation in Lemma 8.2.

Also we can assume that at least some $L_{v} \subset L$ has $m_{v}=+1$. This will assure us that we are in the case of (i) and (ii) that allow us to use Theorem 4.6.

7.9. Homotopy classes of maps $Y \backslash L \rightarrow S^{1}$ are in bijection with $H^{1}(Y \backslash L, \mathbb{Z})$. It follows from the universal coefficient theorem that $H^{1}(Y \backslash L, \mathbb{Z})=H_{1}(Y \backslash L, \mathbb{Z})^{\vee}$. This bijection $\left[Y \backslash L, S^{1}\right] \rightarrow H_{1}(Y \backslash L, \mathbb{Z})^{\vee}$ is realized by sending $f: Y \backslash L \rightarrow S^{1}$ to $f_{*}: H^{1}(Y \backslash L, \mathbb{Z}) \rightarrow H^{1}\left(S^{1}, \mathbb{Z}\right)=\mathbb{Z}$. The associated multiplicities are defined as $m_{w}=f_{*}\left(\left[S_{w}\right]\right)$ for any $w \in \mathcal{W}$.

Note that if the orbifold intersection matrix $I^{\circ}$ is invertible, then eq. (7.4) shows that the family $\left(m_{n}\right)_{n \in \mathcal{N}}$ is determined by $\left(m_{a}\right)_{a \in \mathcal{A}}$.

Proposition 7.10. Let $\gamma: H_{1}(Y \backslash L, \mathbb{Z}) \rightarrow \mathbb{Z}$ be a linear map. The corresponding element of $\left[Y \backslash L, S^{1}\right]$ contains a horizontal fibration if and only if, with notation as above, $m_{n} \neq 0$ for all $n \in \mathcal{N}$. In this case, the fibration is unique up to isotopy.

Before proving the proposition, we state the following lemma.

Lemma 7.11. Let $(Y, L)$ be a Waldhausen link with plumbing graph $\Lambda$. Let $E=$ $Y / \sim$ be the quotient space under the finest equivalence relation $\sim$ so that any Seifert 
fiber is contained in an equivalence class. Define the group $K$ as the set of formal sums as in the left hand side of eq. (7.7). We then have an exact sequence

$$
0 \rightarrow K \rightarrow \mathbb{Z}\left\langle C_{v} \mid v \in \mathcal{V}\right\rangle \rightarrow H_{1}(Y \backslash L, \mathbb{Z}) \rightarrow H_{1}(E, \mathbb{Z}) \rightarrow 0
$$

Furthermore, $H_{1}(E, \mathbb{Z})$ is a direct sum of free groups $H_{G(Y, L)}$ and $H_{n}, n \in \mathcal{N}$, where

$H_{n}=H_{n}\left(E_{n} / \sim, \mathbb{Z}\right)$, where $E_{n}=Y_{n} / \sim$ is the image of $Y_{n}$ in $E$. We note that this is an $r_{n}$-punctured surface of genus $g_{n}$ where $r_{n}$ is the number of components of $L$ contained in $Y_{n}$. We note that $H_{n}$ is contained in the image of $H_{1}\left(Y_{n}, \mathbb{Z}\right)$ via the inclusion and that $\mathrm{rk} H_{n}=2 g_{n}+\max \left\{r_{n}-1,0\right\}$.

S $H_{G(Y, L)}=H_{1}(G(Y, L), \mathbb{Z})$, the map $H_{1}(Y \backslash L, \mathbb{Z}) \rightarrow H_{G(Y, L)}$ is obtained by collapsing $Y \backslash L$ onto $G(Y, L)$ in the obvious way.

Proof. This is in [Ném00, Proposition 2.18].

Proof of Proposition 7.10. In the case when $L$ is a union of fibers in a Seifert manifold, this is proved in [Por]. The general case then follows from [EN85, Theorem 4.2] We provide here a proof which follows [EN85, Theorem 4.2] but does not depend on some deep theorems cited there due to the horizontal assumption.

Let $\operatorname{HFib}(Y, L)$ be the set of horizontal fibrations of the complement $Y \backslash L$. Let $H \subset H^{1}(Y \backslash L, \mathbb{Z})$ be the set of cohomology classes $\gamma$ satisfying $\left\langle\gamma, S_{n}\right\rangle \neq 0$ for all $n \in \mathcal{N}$. If $f: Y \backslash L \rightarrow S^{1}$ is horizontal, then each fiber is transverse to the boundary of the Seifert pieces of $Y \backslash L$. In particular, $f$ restricted to any Seifert piece is a fibration. It follows from [Por] that the general fiber has nonzero intersection with any fiber of $f$, i.e. $m_{n} \neq 0$. In particular, the correspondence $f \mapsto f_{*}$ induces a $\operatorname{map} \operatorname{HFib}(Y, L) \rightarrow H$. We will construct a map $H \rightarrow \operatorname{HFib}(Y, L), \gamma \mapsto f_{\gamma}$ and show that the two maps are inverse to each other.

Let $\gamma \in H$ and set $\gamma_{n}=\left.\gamma\right|_{Y_{n}}$ for $n \in \mathcal{N}$. As $m_{n} \neq 0$, we know that there exists a fibration $f_{n}: Y_{n} \backslash L \rightarrow S^{1}$ satisfying $f_{n, *}=\gamma_{n}$, unique up to isotopy. If $T$ is a torus corresponding to an edge connecting $n$ and $n^{\prime}$, then $\left.\gamma_{n}\right|_{T}=\left.\gamma\right|_{T}=\left.\gamma_{n^{\prime}}\right|_{T}$. It follows that we can isotope $f_{n}$ and $f_{n^{\prime}}$ in a neighborhood around $T$ to coincide on $T$, and to glue together to form a fibration $f: Y \backslash L \rightarrow S^{1}$. The fibration $f_{\gamma}$ is obtained by a modification of $f$ explained below.

Let $A$ be a spanning tree for $G(Y, L)$ and let $\mathcal{E}^{A}$ be the set of edges in $G(Y, L)$ not in $A$. For each $e \in \mathcal{E}^{A}$, let $c_{e}$ be the unique simple circuit in $G(Y, L)$ containing only $e$ and edges in $A$ and let $p_{e}: S^{1} \rightarrow Y \backslash L$ be a path visiting the vertices and edges of the circuit $c_{e}$.

We define $f_{\gamma}: Y \backslash L \rightarrow S^{1}$ as follows. For any $e \in \mathcal{E}^{A}$, let $N_{e}$ be a tubular neighborhood around $T_{e}$. Since the normal bundle of $T_{e} \subset Y$ is orientable, it is trivial: $N_{e} \cong T_{e} \times[0,1]$; let $\pi_{e}: N_{e} \rightarrow[0,1]$ be the projection. We can assume that the intersection $N_{e} \cap p_{e}\left(S^{1}\right)$ is a fiber of the projection onto $T_{e}$, with the orientations coinciding. Define $h_{e}=p_{e, *}\left(\left[S^{1}\right]\right) \in H_{1}(Y \backslash L, \mathbb{Z})$. Using a $\mathcal{C}^{\infty}$ function $\psi:[0,1] \rightarrow \mathbb{R}$, taking constant values 0,1 in neighborhoods around 0,1 , respectively, define

$$
f_{\gamma}=f \cdot \prod_{e \in \mathcal{E}^{A}} e^{2 \pi i\left(\left\langle\gamma, h_{e}\right\rangle-f_{*}\left(h_{e}\right)\right) \psi \circ \pi},
$$

where, outside $N_{e}$, the factor corresponding to $e$ is understood to take the value 1 .

It follows from Lemma 7.11 that the quotient of $H_{1}(Y \backslash L, \mathbb{Z})$ modulo the images of $H_{1}\left(Y_{n} \backslash L, \mathbb{Z}\right)$ for $n \in \mathcal{N}$ is freely generated by the classes $h_{e}$ for $e \in \mathcal{E}^{A}$. By 
construction, $f_{*}, f_{\gamma, *}$ and $\gamma$ coincide on these images. It then follows by the above modification of $f$ that $f_{\gamma, *}\left(h_{e}\right)=\gamma\left(h_{e}\right)$ for all $e \in \mathcal{E}^{A}$, thus $f_{*, \gamma}=\gamma$.

Finally, we must show that, given an $f \in \operatorname{HFib}(Y, L)$, the maps $f$ and $f_{f_{*}}$ are isotopic (where $f_{f_{*}}$ is constructed from eq. (7.12) by taking $\gamma=f_{*}$ ). As above, for all edges $e$ in $G(Y, L)$, the torus $T_{e}$ is transverse to all fibers of $f$. Then the restrictions of $f$ and $f_{f_{*}}$ to any Seifert piece are horizontal. They are therefore, isotopic by [Por], since $\left(f_{f_{*}}\right)_{*}=f_{*}$. Thus, we may assume that $f$ and $f_{f_{*}}$ coincide outside $\cup_{e \in \mathcal{E}^{A}} N_{e}$. Thus, up to isotopy, we may assume that $f / f_{f_{*}}$ equals 1 outside $\cup_{e} N_{e}$, whereas, for an oriented edge $e$, we have $f / f_{f_{*}}=e^{2 \pi i t_{e} \psi \circ \pi}$ on $N_{e}$ for some $t_{e} \in \mathbb{Z}$

Let $\pi$ be a map obtained by collapsing $Y_{n} \backslash \cup_{e} N_{e}$ to a point for all $n \in \mathcal{N}$, as well as collapsing the fibers of $\pi_{e}$ to points. We thus get a map $\pi: Y \backslash L \rightarrow G(Y, L)$. Since $G(Y, L)$ is a graph and $\left(\left(f / f_{f_{*}}\right) \circ \pi\right)_{*}=0$, there is an $h: G(Y, L) \times[0,1] \rightarrow S^{1}$ satisfying $h(\cdot, 0)=\left(f / f_{f_{*}}\right) \circ \pi$ and $h(\cdot, 1)=1$. By multiplication with $h$, we obtain an isotopy between $f$ and $f_{f_{*}}$.

\section{DESCRIPTION OF THE MONODROMY}

The previous sections classifies horizontal fibrations on graph manifolds. However, this does not give us a good understanding of the action of the monodromy on the pages of the open book. This sections is devoted to understand the monodromy and produce numerical data that help us construct the desire mixed tête-à-tête graph in Section 9.

In [LP05, Lemma 3.1] I. Luengo and A. Pichon include a dictionary between horizontal fibrations of Waldhausen links and Nielsen graphs. In this section we start with a horizontal open book of a graph manifold and explain how to get the data of the pseudo-periodic diffeomorphism corresponding to the monodromy in order to produce a mixed tête-à-tête graph whose mapping torus recovers the original graph manifold.

The monodromy may be understood by getting a good grasp of:

(i) The action of the monodromy on the periodic pieces, that is, the number of connected components, the period and the non-trivial isotropy points of each orbit.

(ii) The attaching of the different orbits along annuli.

(iii) The screw numbers of the annuli connecting the periodic pieces.

(iv) The fractional Dehn twist coefficients associated to the components $L_{v}$ of the binding of the open book.

The above information allows us to give a model of the page and a pseudoperiodic automorphism of it in a canonical form. Then, we can apply the procedure described in the proof of Theorem 6.7 to build a mixed tête-à-tête graph modeling the automorphism.

i) Periodic pieces. Let $Y_{j}$ be a Seifert piece. The map $\left.f\right|_{Y_{j}}$ induces an element $\left(\left.f\right|_{Y_{j}}\right)_{*}$ of $H^{1}\left(Y_{j} ; \mathbb{Z}\right)$ that does not vanish on a generic fiber. Hence we can apply the algorithms described in [Por, Section 6.1] to get a description of the topology of the horizontal surface $\Sigma_{j}$ and the monodromy acting on it.

ii)Attaching of orbits along annuli. In order to recover the topology of the page $\Sigma$ we have to glue some boundary components of the periodic pieces $\Sigma_{j}$ constructed above. 
We follow the notation of the second part of Proposition 7.10. For each periodic orbit $\Sigma_{j}$ choose a connected component and denote it by $\Sigma_{j, 1}$. Label the rest according to the action of the monodromy by $\Sigma_{j, 2}, \ldots, \Sigma_{j, \alpha_{j}}$.

Let $A$ be a spanning tree of $G(Y, L)$. First we construct the part of the page $\Sigma$ contained in the pieces of the spanning tree. For each edge $e$ in $A$ connecting vertices $j, j^{\prime}$ there corresponds an orbit of annuli $\mathcal{A}_{e}$ of the page $\Sigma$. This orbit of annuli is in a thickened torus $N_{e}$. Note that since $A$ is a tree, $j \neq j^{\prime}$ and in particular this orbit is not amphidrome.

We glue a boundary component of $\Sigma_{j, 1}$ in $N_{e}$ to a boundary component of $\Sigma_{j^{\prime}, 1}$ in $N_{e}$. We glue the rest of boundary components in that torus equivariantly by the monodromy. Since $A$ is a tree, different choices produce the same surface.

Let $\mathcal{E}^{A}$ be the set of edges in $G(Y, L)$ not in $A$. For each edge $e$ in $\mathcal{E}^{A}$, let $c_{e}$ be the circuit in $G(Y, L)$ that only intersects $\mathcal{E}^{A}$ in $e$. By choosing an inverse to the last arrow of the exact sequence in Lemma 7.11 we get an splitting

$$
H_{1}(Y \backslash L ; \mathbb{Z}) \simeq H_{1}(E ; \mathbb{Z}) \oplus \frac{\mathbb{Z}\left\langle C_{v} \mid v \in \mathcal{V}\right\rangle}{K} .
$$

Which also by Lemma 7.11 factorizes in

$$
H_{G(Y, L)} \oplus \frac{\mathbb{Z}\left\langle C_{v} \mid v \in \mathcal{V}\right\rangle}{K} \bigoplus_{n \in \mathcal{N}} H_{n}
$$

Then $f_{\star}\left(c_{e}\right)$ is a number $n_{e}$ which, up to the choice of splitting, is well defined modulo the greatest common divisor of $\alpha_{j^{\prime \prime}}$ for $j^{\prime \prime}$ on the circuit $c_{e}$. Then, a boundary component of $\Sigma_{j, 1}$ in $N_{e}$ is glued to a boundary component of $\Sigma_{j^{\prime}, 1+n_{e}}$.

Remark 8.1. The number $n_{e}$ contains equivalent data as the action of the monodromy in the partition graph in [MM11] or the invariant $\omega(h)$ in [Pic01].

This completes the construction of the page $\Sigma$.

iii) Screw numbers. We recall that we can easily get the plumbing graph $\Lambda$ from the Waldhausen graph $G(Y, L)$.

Lemma 8.2. Let $n, n^{\prime} \in \mathcal{N}$ be joined by an edge e in $G(Y, L)$. Let $\alpha_{e} / \beta_{e}=$ $\left[b_{1}, \ldots, b_{s}\right]$ be the negative continued fraction expansion. Define $m_{0}, m_{1}, \ldots, m_{s+1}$ by

$$
m_{0}=m_{n}, \quad m_{1}=\frac{\beta_{e} m_{n}+m_{n^{\prime}}}{\alpha_{e}}, \quad m_{i-1}-b_{i} m_{i}+m_{i+1}=0 \quad i=1, \ldots, s .
$$

The screw number $r_{e}$ associated to the edge $e$ in the Nielsen graph of $\phi$ is

$$
-d^{2} \sum_{i=0}^{s} \frac{1}{m_{i} m_{i+1}} \text {. }
$$

where $d=\operatorname{gcd}\left(m_{0}, m_{1}\right)$ which is equal to $\operatorname{gcd}\left(m_{i}, m_{i+1}\right)$ for all $i$.

Proof. Let $A_{1}, \ldots, A_{k}$ be the annuli in $\Sigma$ corresponding to the edge $e$ and let $v_{1}, \ldots, v_{s}$ be the vertices on the bamboo joining $n, n^{\prime}$ in $\Lambda$. The annulus $A_{j}$ can be split up into a union of annuli as

$$
A_{j}=A_{j}^{0,1} \cup A_{j}^{1} \cup A_{j}^{1,2} \cup A_{j}^{2} \cup \cdots \cup A_{j}^{s} \cup A_{j}^{s, s+1} .
$$

Here, $A_{j}^{i, i+1}$ corresponds to the edge joining $v_{i}$ and $v_{i+1}$, and $A_{j}^{i}$ is the complement of these annuli inside the plumbed piece corresponding to $v_{i}$. We can assume that 
$\phi$ is truly periodic on the pieces $A_{j}^{i}$, so $r_{e}$ is the sum of screw numbers associated to the edges connecting $v_{i}$ and $v_{i+1}$ (where we set $v_{0}=n$ and $v_{s+1}=m_{s+1}$ ). This reduces the proof to the case when $\alpha_{e}=1$ and $s=0$.

Denote by $\phi^{\prime}$ the restriction of $\phi^{k}$ to $A_{1}$. It follows from Definition 3.14 that $\phi$ and $\phi^{\prime}$ have the same screw number on $A_{1}^{0,1}$. This reduces the proof to the case when $\operatorname{gcd}\left(m_{0}, m_{1}\right)=1$.

The annulus $A=A_{1}^{0,1}$ is the Milnor fiber of the nonisolated plane curve singularity given by $x^{m_{0}} y^{m_{1}}=0$. Let $F$ be this Milnor fiber and let $F_{\text {sing }}$ be the singular Milnor fiber. In fact, define the total space of the singular Milnor fibration as the closure of the set

$$
\left\{(z, x) \in S^{1} \times S^{3}|f(x) /| f(x) \mid=z\right\}
$$

The singular fibration is the first projection. Furthermore, the singular fibration contains the trivial fibration with fiber $L_{f}$ given by $f(x, y)=x^{m_{0}} y^{m_{1}}=0$. We get the singular monodromy, well defined up to homotopy, $h_{\text {sing }}: F_{\text {sing }} \rightarrow F_{\text {sing }}$, satisfying $\left.h_{\text {sing }}\right|_{L_{f}}=\mathrm{id}_{L_{f}}$.

Note that the oriented real blowup of the singular fiber space along $L_{f}$ is the Milnor fiber. The singular monodromy lifts to a monodromy $h$ on the Milnor fiber. Although this monodromy is not the identity on the boundary, it gives a well defined screw number. In fact, this representative of the monodromy coincides with $\phi$ on $A=F$.

Next, we describe the singular monodromy for the plane curve $f(x, y)=x^{a} y^{b}$, assuming $\operatorname{gcd}(a, b)=1$. Define an action of $\mathbb{R}$ on $S^{3}$ by setting

$$
t *(x, y)=\left(e^{2 \pi i t|y| / a} x, e^{2 \pi i t(1-|y|) / b} y\right), \quad t \in \mathbb{R} .
$$

Note the similarity to the classical computation in Milnor's book for weighted homogeneous polynomials. Since this polynomial is weighted homogeneous for any weight, we can vary the weights. This is done in order to guarantee that the action is trivial on the link $L_{f}$. The link consists of points $(x, y)$ where either coordinate vanishes. Note that we choose a Milnor ball of radius 1 . It follows that this action acts trivially on $L_{f}$. Furthermore, we have $f(t *(x, y))=e^{2 \pi i t} f(x, y)$. It follows that the singular monodromy is obtained by acting by 1 . We parametrize the Milnor fiber by

$$
[0,1] \times S^{1} \rightarrow S^{3}, \quad(s, \eta) \mapsto \frac{\left(\eta^{b} s, \eta^{-a}(s-1)\right)}{\sqrt{s^{2}+(s-1)^{2}}} .
$$

Writing $r(s)^{-1}$ for the denominator, the action of $t=a b$ on this image is

$$
\begin{aligned}
r(s)\left(\eta^{-b} s, \eta^{a}(1-s)\right) & \mapsto r(s)\left(e^{2 \pi i(1-s) b} \eta^{b} s, e^{2 \pi i a} \eta^{-a}(1-s)\right) \\
& =r(s)\left(\left(e^{-2 \pi i s} \eta\right)^{b} s,\left(e^{-2 \pi i s} \eta\right)^{-a}(1-s)\right),
\end{aligned}
$$

i.e. $(s, \eta) \mapsto\left(s, e^{-2 \pi i s} \eta\right)$, a right handed Dehn twist. It follows that the screw number of $\phi$ is $-1 / a b$.

Remark 8.3. Note that the lemma above recovers [MM11, Theorem 5.1 (5)], which is a number theory result, by using a singularity theoretic point of view and a purely topological technique.

Observe that one can also apply the technique of the previous Lemma to part $i$ ). In fact, by applying the lemma to an arm of the plumbing graph, we get 
Corollary 8.4. Let $m_{0}, m_{1}, \ldots, m_{s}, m_{s+1}$ be the multiplicities of an arm of $\Lambda$ corresponding to a special fiber of a Seifert piece. Then, the corresponding rotation number of the non-trivial isotropy orbit is

$$
d^{2} \sum_{i=0}^{s} \frac{1}{m_{i} m_{i+1}} .
$$

iv) fractional Dehn twists at the boundary components fixed by the monodromy. A $L_{v} \subset L$ has $m_{v}=+1$, so there exists a well defined representative of the monodromy fixing the corresponding boundary component of the fiber near $L_{v}$. Then, it makes sense to compute the fractional Dehn twist coefficient at that boundary component.

We observe that fractional Dehn twist coefficients can be seen as screw numbers. Let $C$ be a boundary component of a periodic part $\Sigma_{j}$ the fiber. Suppose that $\left.\phi\right|_{C}=$ id. We can glue an annulus $A$ to this boundary component and extend $\phi$ by the identity to all the annulus. Then the fractional Dehn twist of $\phi$ at $C$ coincides with minus the screw number of $\phi$ between the periodic piece $A$ and the periodic piece $\Sigma_{j}$. Hence, from Lemma 8.2 we get the following

Corollary 8.5. Let $m_{0}, m_{1}, \ldots, m_{s}, m_{s+1}$ be the multiplicities of an arm of $\Lambda$ ending at an arrowhead (thus $m_{s+1}=1$ ). Then, the corresponding fractional Dehn twist coefficient is

$$
d^{2} \sum_{i=0}^{s} \frac{1}{m_{i} m_{i+1}}
$$

By the fact that all multiplicities are positive and the edges have weight +1 (recall 7.8), we get that these fractional Dehn twist coefficients are positive which allows the monodromy to be modeled by a mixed tête-à-tête graph.

\section{From HORIZONTAL OPEN BOOKS TO MIXED TÊTE-À-TÊTE GRAPHS}

Suppose we are given:

(i) A graph manifold $Y$ given by a plumbing graph $\Lambda$.

(ii) A Waldhausen link $L \subset Y$.

(iii) A homomorphism $\gamma: H_{1}(Y \backslash L, \mathbb{Z}) \rightarrow \mathbb{Z}$ that doesn't vanish on the classes of generic Seifert fibers of all Seifert pieces and whose associated system of multiplicities satisfies that $m_{v}=+1$ for all components $L_{v}$ of $L$.

Section 8 provides the combinatorial data associated with the monodromy $\phi$ of the fibration $f$ so that $f_{*}=\gamma$. Now, Theorem 6.7 produces a mixed têteà-tête graph inducing $\phi$. This construction is made explicit in the proof of the theorem. This is done by first choosing relative tête-à-tête graphs for those pieces in $Y$ which contain a component of $L$. An iterative process follows: choose an invariant spine of each neighboring piece; then the screw number of each orbit of connecting annuli produces a parametrization of these annuli and some retraction lines of these parametrizations are used to connect the previously constructed graph with the new invariant spines. This produces the desired mixed tête-à-tête graph. 


\section{FROM MIXED TÊTE-À-TÊTE GRAPHS TO OPEN BOOKS}

In this section we describe an algorithm that works in the reverse direction of the previous algorithm. That is, we start with a mixed tête-à-tête graph $\left(\Gamma^{\bullet}, \delta_{\bullet}\right)$ with a thickening $\Sigma$, and the algorithm produces:

(i) A graph manifold $Y$ represented by a plumbing graph $\Lambda$

(ii) A Waldhausen link $(Y, L)$ such that $Y \backslash L$ is diffeomorphic to the mapping torus of the mixed tête-à-tête automorphism $\phi_{\Gamma}$.

(iii) An element $f_{*} \in H^{1}(Y \backslash L ; \mathbb{Z})$ given by a horizontal open book $f: Y \backslash L \rightarrow$ $\mathbb{S}^{1}$ that has fiber diffeomorphic to $\Sigma$ and whose monodromy is conjugate to that of $\phi_{\Gamma}$.

First we are going to obtain the information about the Seifert pieces of $Y$. Recall that $\left.\phi_{\Gamma^{,}, i}\right|_{\Gamma_{\Gamma^{i+1}}^{i}}: \Gamma_{\Gamma^{i+1}}^{i} \rightarrow \Gamma_{\Gamma^{i+1}}^{i}$ is a periodic automorphism. For each $p \in \Gamma_{\Gamma^{i+1}}^{i} \subset$ $\Gamma_{\Gamma^{i+1}}$ we recall that $\phi_{\Gamma, i}(p)$ is, by definition, the last point of the walk resulting from the concatenation $\gamma_{p}^{i} \star \cdots \star \gamma_{p}^{0}$.

Let $\ell$ be the depth of the filtration of $\Gamma^{\bullet}$. For each $i=0, \ldots, \ell$ we have that the graph $\Gamma_{\Gamma^{i+1}}^{i}$ and the restriction $\left.\phi_{\Gamma, i}\right|_{\Gamma_{\Gamma^{i+1}}^{i}}$ give us

(i) The number of Seifert pieces that lie on the $i$-th level of the filtration. This is the number of orbits of $\phi_{\Gamma, i}$ on the set of connected components of $\Gamma_{\Gamma^{i+1}}^{i}$. For each Seifert piece, there will be a node on $\Lambda$.

(ii) The number of connected components in each orbit.

(iii) The multiplicity $m_{n}$ corresponding to the node $n$ of the corresponding Seifert piece which is the order of $\left.\phi_{\Gamma, i}\right|_{\Gamma_{\Gamma^{i} i+1}^{i}}$ restricted to the corresponding orbit.

(iv) By applying Steps 1-4 of the algorithm From general tête-à-tête graphs to star-shaped plumbing graphs in [Por], we get the decoration corresponding to the Euler numbers of the arms of each Seifert piece.

Note that we leave the nodes without the Euler number decoration until the end.

10.1. We recall a piece of the algorithm in [Por] which will be useful now. Suppose that $\left.\phi_{\Gamma, i}\right|_{\Gamma_{\Gamma^{i+1}}^{i}}$ has order $n$. If $x \in \Gamma_{\Gamma^{i+1}}^{i}$ is a point with non-trivial isotropy in $\left\langle\left.\phi_{\Gamma, i}\right|_{\Gamma_{\Gamma^{i+1}}^{i}}\right\rangle$, then there exists a natural number $0<k<n$ such that $\left.\phi_{\Gamma_{,}, i}\right|_{\Gamma_{\Gamma^{i}+1}^{i}} ^{k}(x)=$ $x$ and in this case, $\left.\phi_{\Gamma, i}\right|_{\Gamma_{\Gamma^{i+1}}^{i}} ^{k}$ is a rotation in a disk around $x$. Suppose that this rotation has rotation number $p / q$ with $q=n / k$. The rotation number is measured at the boundary of the disk with the orientation induced as boundary of the disk. Then the Seifert fiber over $x$ has Seifert invariants $(\alpha, \beta)=(q,-b)$ with $b p \equiv 1 \bmod q$ and $0<-b<q$. By [Por, Lemma 3.2] and discussion before in that same work, the attaching matrix is

$$
([s][f])=([m][l])\left(\begin{array}{ll}
a & p \\
b & q
\end{array}\right)
$$

where $[s],[f]$ (section, fiber) is a positive basis of the boundary torus in the circle bundle of the corresponding node; and $[m],[l]$ (meridian, longitude) is a basis on the boundary torus of the solid torus $D^{2} \times \mathbb{S}^{1}$ that is attached. Each of the boundaries of the $k$ disks is glued to a meridian of this solid torus. By extending the fibration on the node to the attached solid torus, we get a $(p, q)$-fibered torus which induces a rotation with rotation number $p / q$ on each of the $k$ boundary components. 
The decoration of the Euler numbers in the arm corresponding to that Seifert fiber is given by the continued fraction of $q /-b$.

10.3. Now we describe the Euler weights of the arms of the plumbing graph that end at arrowheads with multiplicity (1). These correspond to boundary components of $\Sigma$ that are fixed by $\phi_{\Gamma}$. Let $p / q$ be the fractional Dehn twist at a boundary component (note that possibly $p>q$ ). Let $b$ be as before, i.e. $b p \equiv 1 \bmod q$ and $0<-b<q$. Then the Euler numbers of the vertices of the arm correspond to the continued fraction expansion of $-p / a$ with $a=(p b-1) / q$. The reason is that by the construction of an open book, we need to attach a solid torus $D^{2} \times \mathbb{S}^{1}$ to the mapping torus of $\phi_{\Gamma}$ matching $p t \times \mathbb{S}^{1}$ with the boundary of the surface. This corresponds to change the matrix from 10.2 to

$$
\left(\begin{array}{ll}
0 & 1 \\
1 & 0
\end{array}\right)\left(\begin{array}{ll}
a & p \\
b & q
\end{array}\right)=\left(\begin{array}{ll}
b & q \\
a & p
\end{array}\right)
$$

Hence, by using [Por, Lemma.34], we have that the decoration of the Euler numbers in the arm corresponding to that Seifert fiber is given by the continued fraction expansion of $-p / a$. The arm ends with an arrow with multiplicity weight equal to $(1)$.

10.5. Finally we describe the bamboos joining nodes in the plumbing graph. To each orbit of annuli in the almost-canonical form defined by a mixed tête-à-tête graph there corresponds a bamboo in the plumbing graph. [FPP, Remark 8.12] gives us a simple formula for the screw number associated with an orbit of annuli that corresponds to components $\tilde{\Gamma}_{j, 1}^{i}, \ldots, \tilde{\Gamma}_{j, \alpha_{j}}^{i}$ of $\tilde{\Gamma}^{i}$ (recall construction [FPP]). We have that the screw number is $\sum_{k}-\delta_{j, k}^{i} / l\left(\tilde{\Gamma}_{j, 1}^{i}\right)$.

The maps $\left.\phi_{\Gamma, i}\right|_{\Gamma_{\Gamma^{i+1}}^{i}}$ and $\left.\phi_{\Gamma, i+1}\right|_{\Gamma_{\Gamma^{i+2}}^{i+1}}$ give us the rotation numbers of $\phi_{\Gamma}^{\alpha_{j}}$ restricted to each boundary component of the annuli $\mathcal{A}_{j, 1}^{i}$. These rotation numbers are $p_{0} / q_{0}$ and $p_{1} / q_{1}$. The screw number can then be written as $1-p_{0} / q_{0}-p_{1} / q_{1}+t$ with $t \in \mathbb{Z}$. Then, the gluing matrix from the Seifert piece corresponding to the node $n_{0}$ to the Seifert piece corresponding to the node $n_{1}$ is the composition of three matrices. The first matrix corresponds to performing a twist of fractional Dehn twist coefficient equal to $p_{0} / q_{0}-1-t$ near the boundaries of $n_{0}$; the second matrix $\left(\begin{array}{cc}-1 & 0 \\ 0 & 1\end{array}\right)$ represents the gluing of boundary components from $n_{0}$ to boundary components in $n_{1}$ (the -1 on the top left is because boundaries inherit opposite orientations); and the last matrix has the same meaning as the first, but the remaining amount of twisting is $p_{1} / q_{1}$. Hence, the gluing matrix is:

$$
\begin{gathered}
\left(\begin{array}{ll}
a_{1} & p_{1} \\
b_{1} & q_{1}
\end{array}\right)^{-1}\left(\begin{array}{cc}
-1 & 0 \\
0 & 1
\end{array}\right)\left(\begin{array}{cc}
a_{0} & p_{0}-(t+1) q_{0} \\
b_{0} & q_{0}
\end{array}\right)= \\
=\left(\begin{array}{cc}
q_{1} a_{0}+p_{1} b_{0} & q_{1}\left(p_{0}-(t+1) q_{0}\right)+p_{1} b_{0} \\
* & *
\end{array}\right)
\end{gathered}
$$

Where $b_{1} p_{1} \equiv 1 \bmod q_{1}$ and $0<-b_{1}<q_{1}$; and $b_{0}\left(p_{0}-(t+1) q_{0}\right) \equiv 1 \bmod q_{0}$ and $0<-b_{0}<q_{0}$. And numbers $a_{0}$ and $a_{1}$ are defined by the property that the matrices have determinant -1 . If $q_{i}=1$, then we take $b_{i}=0$ and hence $a_{i}=-1$, for $i=0,1$. 
Hence, again using [Por, Lemma 3.4] we have that the Euler number weights corresponding to the bamboo are given by the continued fraction expansion of

$$
-\frac{q_{1}\left(p_{0}-(t+1) q_{0}\right)+p_{1} b_{0}}{q_{1} a_{0}+p_{1} b_{0}} .
$$

In case the denominator is 0 , this correspond to an empty bamboo or just and edge joining the two nodes.

Summarizing we already have a plumbing graph $\Lambda$ of which we know

(i) Genus number for all vertices.

(ii) The Euler number of all the vertices in the bamboos.

(iii) The multiplicities at the nodes and the arrowheads.

Since we know the Euler numbers of the bamboos, the multiplicities everywhere and that the multiplicities at the nodes are non-zero, we can recover the Euler number weights of the nodes using Equation (7.7). Which completes all the information about the plumbing graph.

Note that this a plumbing graph with arrows, so there is an associated closed graph manifold coming from forgetting the arrows. This is $Y$ and the arrows correspond to the Waldhausen link $L$.

The multiplicities at the nodes together with the Euler number weights, determine a system of multiplicities on the whole graph. However, this information does not, in general, fully determine a horizontal fibration.

We also know the number of connected components of each periodic part. The only piece of information missing to recover the horizontal open book up to diffeomorphism, is the invariant defined Section $8 \mathrm{ii}$ ). That is, we need to give an element on $H_{G(Y, L)}^{\vee}$. Let $c$ be a cycle in $H_{G(Y, L)}$, pick a path $p_{c}$ in $\Gamma$ which is a lift of $c$. There exists a number $k_{c}$ such that the image by $\phi_{\Gamma}^{k_{c}}$ of end point of $p_{c}$ is in the same connected component as the starting point of $p_{c}$. This is the missing invariant.

\section{EXAMPLES}

Example 11.1. Let $\Sigma$ be the surface of Figure 11.1. Suppose it is embedded in $\mathbb{R}^{3}$ with its boundary component being the unit circle in the $x y$-plane. Consider the rotation of $\pi$ radians around the $z$-axis and denote it by $R_{\pi}$. By the symmetric embedding of the surface, it leaves the surface invariant. Isotope the rotation so that it is the identity on $z \leq 0$ and it has fractional Dehn twist coefficient equal to $1 / 2$. We denote the isotoped automorphism by $T$. 


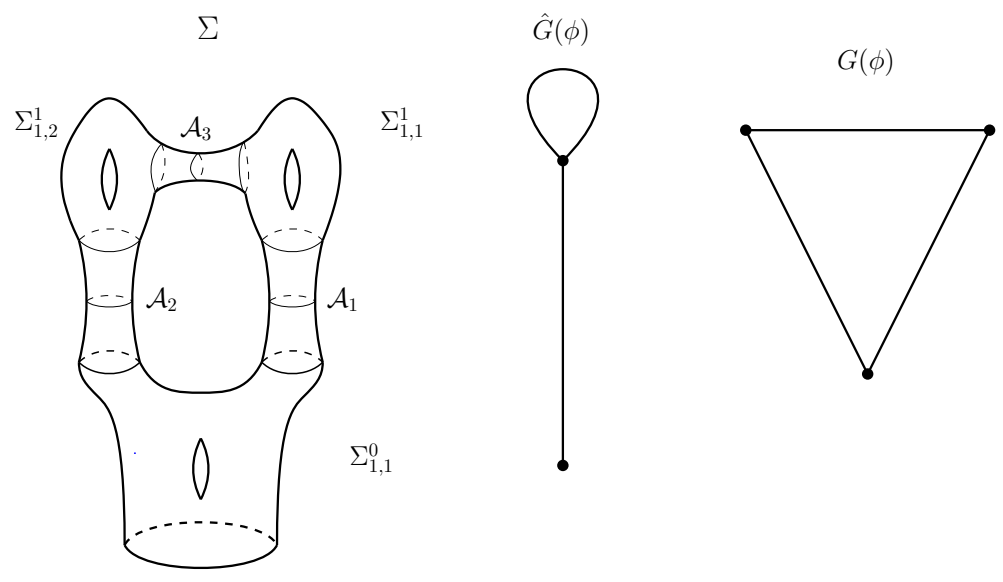

Figure 11.1. On the left we see the surface $\Sigma$. On the right we see the corresponding graphs $\hat{G}(\phi)$ and $G(\phi)$ for the depicted canonical form.

More concretely, let $T: \mathbb{R}^{3} \rightarrow \mathbb{R}^{3}$ defined by

$$
T(r, \theta, z):=\left\{\begin{array}{ccc}
\left(r e^{i(\theta+\pi)}, z\right) & \text { if } & z \leq \varepsilon \\
\left(r e^{\theta+\frac{z}{\varepsilon} \pi}, z\right) & \text { if } & 0 \leq z \leq \varepsilon \\
i d & \text { if } & z \leq 0
\end{array}\right.
$$

With $(r, \theta)$ polar coordinates on the $x y$-plane and $\varepsilon>0$ small.

Let $D_{i}$ be a full positive Dehn twist on the annuli $\mathcal{A}_{i}, k=1,2,3$ (See Figure 11.1). We define the automorphism

$$
\phi:=\left.\mathcal{D}_{3}^{-1} \circ \mathcal{D}_{2} \circ \mathcal{D}_{1}^{-2} \circ T\right|_{\Sigma} .
$$

The automorphism comes already in canonical form. We construct the corresponding Nielsen graph $\hat{G}(\phi)$ and we observe that the corresponding distance function $D$ is not a filtering function since there is 1 loop on $\hat{G}(\phi)$. So we apply Remark 6.4 and we get the almost-canonical form and graphs of figure Figure 11.2.

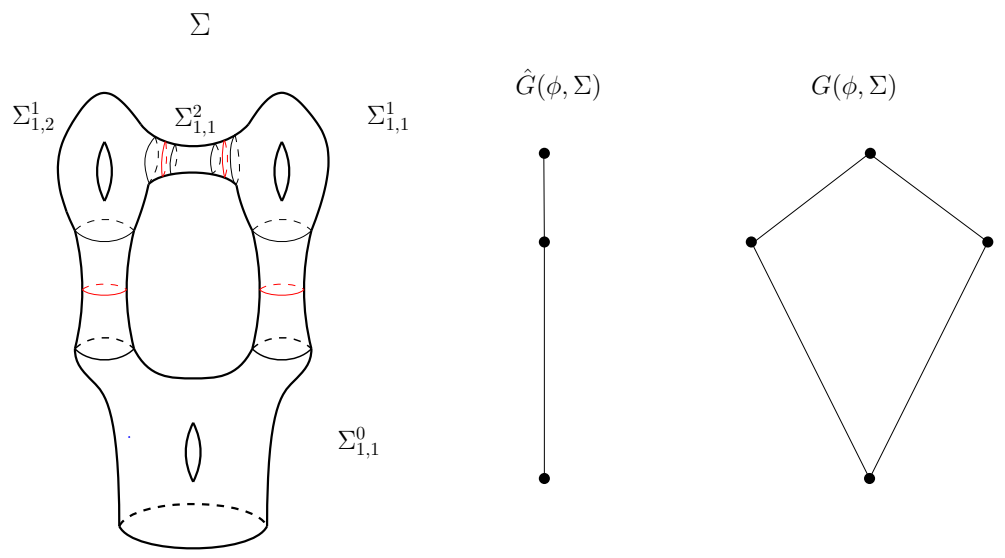

Figure 11.2. On the left we see the surface $\Sigma$. On the right we see the corresponding graphs $\hat{G}(\phi)$ and $G(\phi)$ for the depicted almost-canonical form. In red we see the core curves of the annuli in $\mathcal{A}$. 
Now there are 4 annuli in $\mathcal{A}$ in this almost-canonical form. The annuli $\mathcal{A}_{1}$ and $\mathcal{A}_{2}$ are exchanged by the monodromy, the Dehn twists $\mathcal{D}_{1}^{-2}$ and $\mathcal{D}_{2}$ indicate that the screw number of this orbit is -1 . And the annuli $\mathcal{A}_{3,1}$ and $\mathcal{A}_{3,2}$ that were originally contained in $\mathcal{A}_{3}$; these annuli are also exchanged by the monodromy. We get that the screw number on this orbit is -1 .

We start the construction process following Theorem 6.7. We construct a relative tête-à-tête graph $(\Gamma[0], B[0])$ for $\left.\phi\right|_{\Sigma^{0}}: \Sigma^{0} \rightarrow \Sigma^{0}$. We use [FPP, Theorem 5.22] for this. In Figure 11.3 we can see this graph in blue.

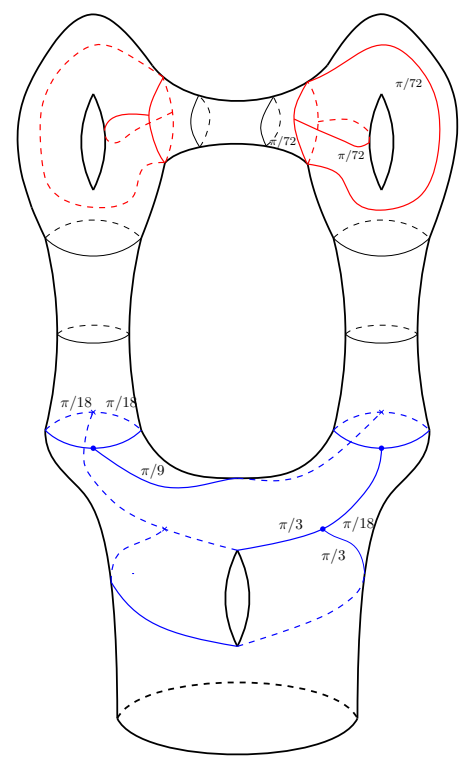

Figure 11.3. The relative tête-à-tête graph $(\Gamma[0], \mathcal{B}[0])$ embedded in $\Sigma^{0} \subset \Sigma$. The lengths are indicated on a few edges and the rest is obtained by symmetry of the graph. We can also see in red an invariant relative spine for $\Sigma^{1}$.

In the next step we construct relative metric ribbon graphs for $\Sigma^{1}$. In this case $\Sigma^{1}$ consist of two connected surfaces that are exchanged. Each surface is a torus with two disks removed and one of the boundary components is glued to an annulus connecting it with $\Sigma^{2}$. This graph will correspond with $\Gamma_{\Gamma^{2}}^{1}$ in the final mixed têteà-tête graph. In the notation of the Theorem we are using, it is $\Gamma[1]^{1}$. In Figure 11.3 we can see these relative metric ribbon graphs in red. In this step we also choose an invariant product structure on $\Sigma_{\Gamma^{1}}^{1}$

Now we proceed to find the parametrizations $\eta_{1}$ and $\eta_{2}$. We pick any parametrization $\eta_{1}$ for $\mathcal{A}_{1}$. On the right part of Figure 11.4 we can see the two retraction lines of the parametrization starting at the two vertices $p$ and $q$ of the corresponding boundary component in $B[0]$. On the left part of that figure we see two annuli, the upper one shows the image of the two retraction lines by $\phi$, on the lower annulus we see the retraction lines that we choose according to Lemma 3.16.

We concatenate the chosen retraction lines of the annuli (green in 11.5) with the corresponding retraction lines of the product structure in $\Sigma^{1}$ (orange in the the picture). 


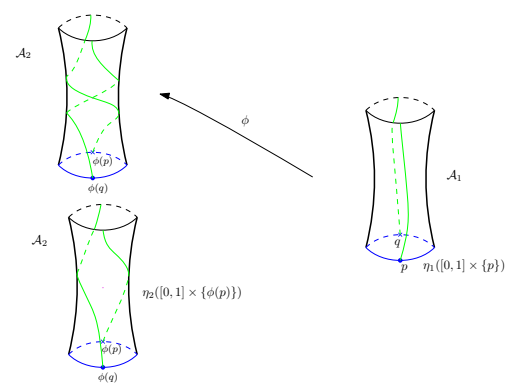

Figure 11.4. The orbit of annuli $\mathcal{A}_{1}$ and $\mathcal{A}_{2}$. On the right part we see $\mathcal{A}_{1}$ with a chosen product structure and on the left part we see two copies of $\mathcal{A}_{2}$, the lower one with the parametrization given by Lemma 3.16.

The metric on the red part is chosen so that each of the two components of $\tilde{\Gamma}^{1}$ has the same length as the two relative components in $\mathcal{B}[0]$, that is equal to $\pi / 9$. Since the screw number is -1 and there are two annuli in the orbit we get that $\delta_{1}$ is the constant function $\pi / 18$.

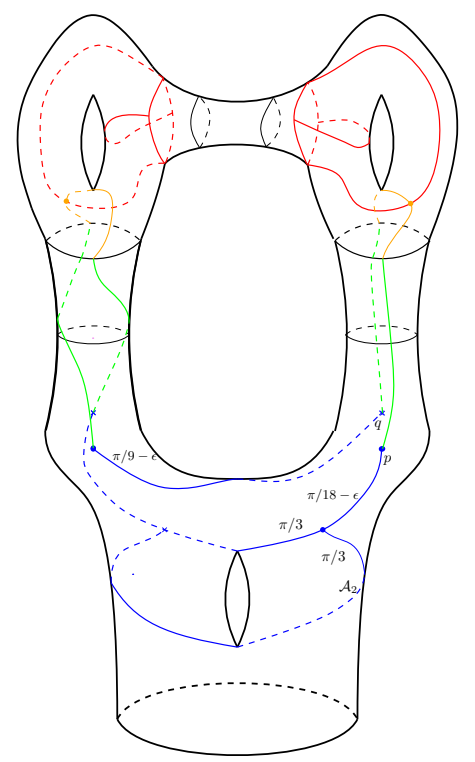

Figure 11.5. The relative tête-à-tête graph $(\Gamma[1], \mathcal{B}[1])$ embedded in $\Sigma^{1} \subset \Sigma$. The lengths are indicated. The green lines correspond to retraction lines of the corresponding product structures $\eta_{1}$ and $\eta_{2}$ and the orange lines correspond to retraction lines of the product structures chosen for the cylinders $\Sigma_{\Gamma[1]^{1}}^{2}$

Similarly to the construction of the graph $(\Gamma[1], \mathcal{B}[1])$, we construct $\Gamma[2]=\Gamma$. We observe that $\Sigma^{2}$ is an annulus whose boundary components are permuted by $\phi$. It is attached along an orbit of annuli $\mathcal{A}_{3,1}$ and $\mathcal{A}_{3,2}$ to $\Sigma^{2}$. It has total length equal to $4 \pi / 72=\pi / 18$ Since this orbit of annuli has screw number $-1 / 2$, we get that $\delta_{2}$ is the constant function $\pi / 144$.

Example 11.3. In this example, we take the mixed tête-à-tête graph $\left(\Gamma^{\bullet}, \delta_{\bullet}\right)$ described in Example 11.1 and compute the corresponding plumbing graph and 


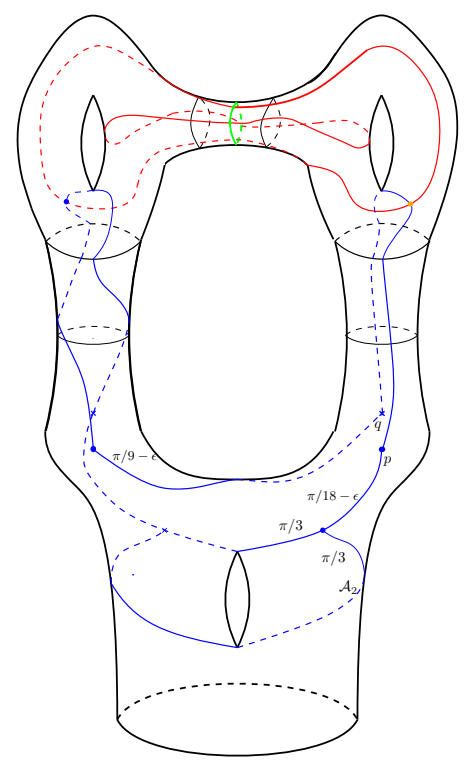

Figure 11.6. The final mixed tête-à-tête graph $\left(\Gamma^{\bullet}, \delta_{\bullet}\right)$. In green we have $\Gamma^{2}$; in red we have $\Gamma^{1} \backslash \Gamma^{2}$ and in blue $\Gamma \backslash \Gamma^{1}$.

associated element of cohomology describing the horizontal surface given by the thickening $\Sigma$ of $\Gamma$. We will use notation from the cited example.

First we observe that since the depth of the graph is 2 , and on each level there is only one orbit of surfaces, the plumbing manifold $Y$ will have 3 Seifert pieces and hence 3 nodes. These correspond exactly to $\Sigma^{0}, \Sigma^{1}$ and $\Sigma^{2}$.

Now we look for the combinatorial information to get the plumbing graph. We observe the following:

(i) The piece corresponding to $\Sigma^{0}$ has three fixed points with rotation number $1 / 2$. The genus of the orbit space is 0 and the automorphism has periodic order 2 restricted to it.

(ii) The boundary has fractional Dehn twist coefficient equal to $1 / 2$.

(iii) The two different orbit of annuli have screw number equal to -1 .

(iv) The Seifert piece corresponding to $\Sigma^{1} \backslash \Sigma^{2}$ has no points with non-trivial isotropy. The genus of the orbit space is 1 and the automorphism has periodic order 2 restricted to it.

(v) The Seifert piece corresponding to $\Sigma^{2}$ has two fixed points with rotation number $1 / 2$. The genus of the orbit space is 0 and the automorphism has periodic order 2 restricted to it.

By using 10.1,10.3 and 10.5 we get the decoration of the plumbing graph. For example blue node has multiplicity 2 , it has three arms coming out of it with only one vertex each with Euler weight -2 (corresponding to the continued fraction of $1 / 2$ ). Since they are fixed points, they have multiplicity weight (1). There is an arrowhead coming out of the blue node corresponding to the only boundary component. The fractional Dehn twist at this boundary component is $1 / 2$ so by using 10.3 we get that the arm ending in an arrowhead has no vertices. Similarly we get the rest of the decoration of the graph. 
Since the graph is contractible, we have that the homology of the Waldhausen graph $H_{1}(G ; \mathbb{Z})=0$ does not contribute to the classification of horizontal fibrations. All the information that's left to determine the horizontal fibration is the number of connected components on each node which is: one connected component on $\Sigma^{0}$, two on $\Sigma^{1}$ and one on $\Sigma^{2}$. In the next example, we analyze the only other possibility of pseudo-periodic automorphism whose mapping torus gives the same plumbed manifold and Waldhausen graph.

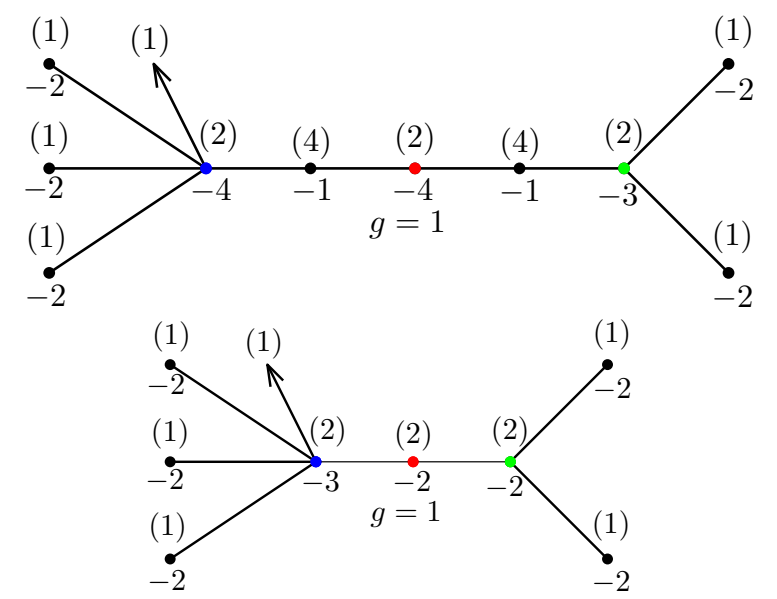

Figure 11.7. The plumbing graph, the colors indicate which Seifert piece they represent according to 11.7. The second figure represents the plumbing graph after plumbing calculus.

Example 11.4. On this example we show an application of the algorithm that takes a 3-dimensional graph manifold with a Waldhausen link and a horizontal fibration as input and returns a mixed tête-à-tête graph as output.

We use the plumbing graph of the previous example Figure 11.7. We consider the same system of multiplicities. The arrowhead on the blue node determines that $\Sigma^{0}$ is connected. On the red node, we have exactly two choices since the gcd of its multiplicity and its neighbors multiplicities is 2 . The previous example corresponds to the horizontal fibration with two connected components forming $\Sigma^{1}$.

In this example, we construct the mixed tête-à-tête graph corresponding to the horizontal open book whose periodic piece corresponding to the red node has one connected component instead of two.

First we observe that since there are no arms coming out of the node, there are no points with non-trivial isotropy. The multiplicity on the node indicates that the periodic automorphism has order 2 . Hence, there is an unramified cover of degree 2 from the piece in the horizontal surface to the orbit space, which is a surface with 2 boundary components and genus 1 . Hence, the piece in the horizontal surface has 4 boundary components and genus 1 . Also, since there are no arms coming out of that vertex, we get that there are no points with non-trivial isotropy.

In Figure 11.8 we see the surface and the periodic automorphism correspnding with the above information. 
$\Sigma^{1}$

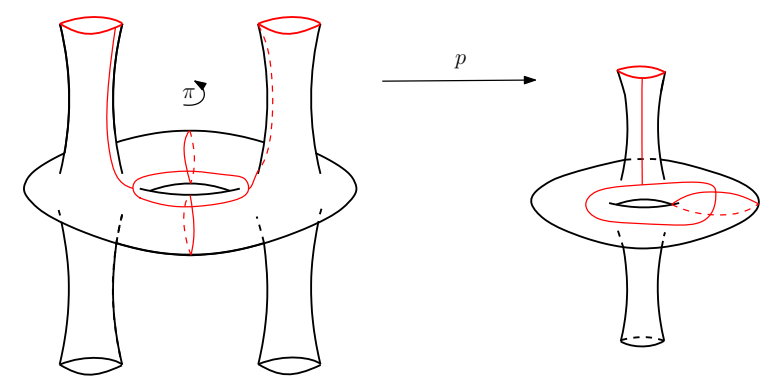

Figure 11.8. On the left we see the surface $\Sigma^{1}$ with the chosen invariant spine by the $\pi$-rotation around the vertical axis. On the right we see the orbit space with a spine whose preimage by the cover $p$ gives the invariant spine.

The surfaces $\Sigma^{0}$ and $\Sigma^{2}$ are exactly the same. Hence, by following again Theorem 6.7 and figure Figure 11.8 we get the following mixed tête-à-tête graph (see Figure 11.9) where the lengths of the edges are indicated in the figure.

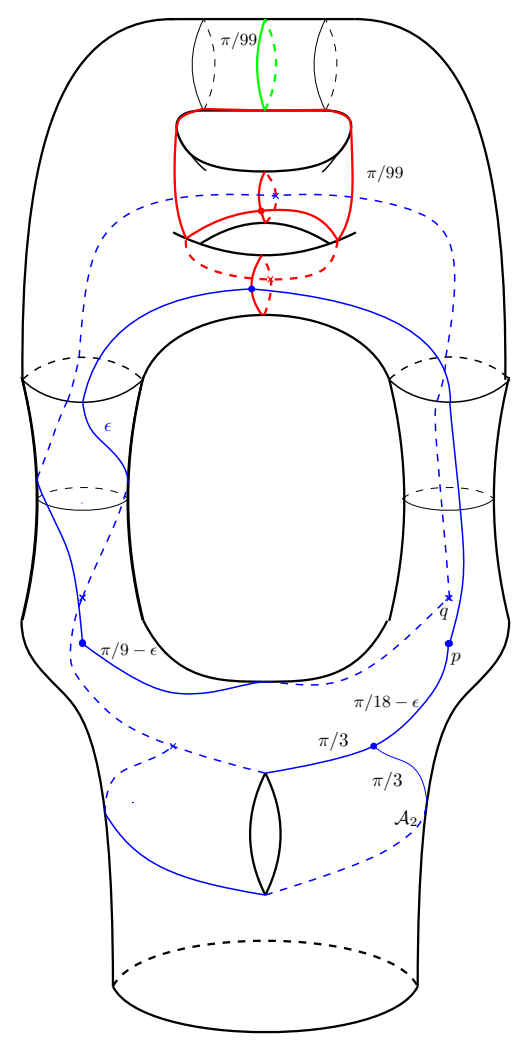

Figure 11.9. The final mixed tête-à-tête graph $\Gamma$. Each red edge has length $\pi / 99$, the green circle also has length $\pi / 99$, the for blue edges that go from $\Sigma^{0}$ to $\Sigma^{1}$ have length $\varepsilon$. The rest of the edges is the same as in the previous example. 
Finally, the $\delta$ functions are $\delta_{0}=\pi, \delta_{1}=\pi / 18$ and $\delta_{2}=\pi / 198$.

\section{REFERENCES}

[A'C] Norbert A'Campo. Tête-à-tête twists and geometric monodromy. Preprint, 2010.

[A'C73] Norbert A'Campo. Sur la monodromie des singularités isolées d'hypersurfaces complexes. Invent. Math., 20:147-169, 1973.

[EN85] David Eisenbud and Walter D. Neumann. Three-dimensional link theory and invariants of plane curve singularities., volume 110 of Annals of Mathematics Studies. Princeton University Press, 1985.

[FM12] Benson Farb and Dan Margalit. A primer on mapping class groups, volume 49 of Princeton Mathematical Series. Princeton University Press, Princeton, NJ, 2012.

[FPP] Javier Fernández de Bobadilla, María Pe Pereira, and Pablo Portilla Cuadrado. Representation of surface homeomorphisms by tête-à-tête graphs. 2017.

[Gra14] Christian Graf. Tête-à-tête graphs and twists. Aug 2014.

[LP05] Ignacio Luengo and Anne Pichon. Lê's conjecture for cyclic covers. In Singularités FrancoJaponaises, volume 10 of Sémin. Congr., pages 163-190. Soc. Math. France, Paris, 2005.

[MM11] Yukio Matsumoto and José María Montesinos-Amilibia. Pseudo-periodic maps and degeneration of Riemann surfaces, volume 2030 of Lecture Notes in Mathematics. Springer, Heidelberg, 2011.

[Ném00] András Némethi. Resolution graphs of some surface singularities. I: Cyclic coverings. In Singularities in algebraic and analytic geometry., pages 89-128. Providence, RI: American Mathematical Society (AMS), 2000.

[Neu81] Walter D. Neumann. A calculus for plumbing applied to the topology of complex surface singularities and degenerating complex curves. Trans. Am. Math. Soc., 268:299-343, 1981.

[Neu97] Walter D. Neumann. Commensurability and virtual fibration for graph manifolds. Topology, 36(2):355-378, 1997.

[Nie44] Jakob Nielsen. Surface transformation classes of algebraically finite type. Danske Vid. Selsk. Math.-Phys. Medd., 21(2):89, 1944.

[NP07] Walter D. Neumann and Anne Pichon. Complex analytic realization of links. In Intelligence of low dimensional topology 2006, Hiroshima, Japan July 22-26, 2006, pages 231-238. Hackensack, NJ: World Scientific, 2007.

[Pic01] Anne Pichon. Fibrations sur le cercle et surfaces complexes. Ann. Inst. Fourier (Grenoble), 51(2):337-374, 2001.

[Por] Pablo Portilla Cuadrado. General tête-à-tête graphs and Seifert manifolds. 2017.

[Wal67] F. Waldhausen. Eine Klasse von 3-dimensionalen Mannigfaltigkeiten. I, II. Invent. Math., 3:308-333, 1967. 